\title{
Parton distributions for event generators
}

\author{
Hung-Liang Lai, ${ }^{a}$ Joey Huston, ${ }^{b}$ Stephen Mrenna, ${ }^{c}$ Pavel M. Nadolsky, ${ }^{d}$ \\ Daniel Stump, ${ }^{b}$ Wu-Ki Tung ${ }^{b, e, 1}$ and C.-P. Yuan $^{b}$ \\ ${ }^{a}$ Taipei Municipal University of Education, \\ Taipei, Taiwan \\ ${ }^{b}$ Department of Physics and Astronomy, Michigan State University, \\ East Lansing, MI 48824-1116, U.S.A. \\ ${ }^{c}$ Fermilab, \\ Batavia, IL 60510 U.S.A. \\ ${ }^{d}$ Department of Physics, Southern Methodist University, \\ 75275 Dallas, TX, U.S.A. \\ ${ }^{e}$ Department of Physics, University of Washington, \\ Seattle, WA 98105, U.S.A. \\ E-mail: laihungl@gmail.com, huston@msu.edu, mrenna@fnal.gov, \\ nadolsky@physics.smu.edu, pumplin@pa.msu.edu, stump@pa.msu.edu, \\ yuan@pa.msu.edu
}

ABSTRACT: In this paper, conventional global QCD analysis is generalized to produce parton distribution functions (PDFs) optimized for use with event generators at the Large Hadron Collider (LHC). This optimization is accomplished by complementing usual constraints on the PDFs from the existing hard-scattering experimental data with those needed to reproduce cross sections for key scattering processes at the LHC, as predicted by the best available theory, in the joint input to the global analysis. With the optimized PDFs, predictions obtained by event generators at a given order in the QCD coupling strength reproduce the representative LHC cross sections computed at one higher order. In the present study, the optimized PDFs for leading-order event generators were developed. Several optimization strategies and resulting candidate PDF sets (labeled as CT09MCS, CT09MC1 and CT09MC2) are compared with those from other approaches.

KEYWords: QCD Phenomenology

ArXiv EPRINT: 0910.4183

\footnotetext{
${ }^{1}$ Deceased
} 


\section{Contents}

1 Introduction 1

2 Global analysis of PDFs for LO event generators 3

$\begin{array}{lll}3 & \text { Impact of parton showering } & 7\end{array}$

4 Results of the modified PDF analysis 18

4.1 General considerations 8

$\begin{array}{llr}4.2 & \text { Numerical results } & 10\end{array}$

$\begin{array}{lll}\text { 4.2.1 CT09MCS PDFs } & 10\end{array}$

$\begin{array}{lll}\text { 4.2.2 } & \text { CT09MC1 and CT09MC2 PDFs } & 11\end{array}$

$\begin{array}{lll}\text { 4.2.3 CT09MC2 predictions for selected LHC cross sections } & 12\end{array}$

5 Comparisons of PDFs 13

6 Predictions for other LHC cross sections $\quad 15$

$\begin{array}{lll}7 & \text { Impact on the underlying event at the LHC } & 17\end{array}$

8 Conclusion $\quad 18$

9 In memoriam $\quad 21$

A Production of a $W$-boson with $n$ partons in the LO-MC approach 22

B K-factors for LO-MC PDFs 24

\section{Introduction}

Monte Carlo event generators play a critical role in all stages of modern particle physics, from detector design to calculation of acceptances and interpretation of experimental results. A key input needed for event generators is parton distribution functions (PDFs). The PDFs are used (1) in the evaluation of the hard subprocess matrix elements, (2) in the backward showering algorithm for initial-state radiation, and (3) in the calculation of the multiple parton interactions that make up the bulk of the underlying event. The latter, in particular, requires extensive tuning which depends strongly on detailed features of the input PDFs.

A long-standing question, and dilemma, in this regard has been: what are the appropriate PDF sets that one should use with the available event generators, in particular, with the most mature and widely used leading-order (LO) generators? For next-to-leading-order 
(NLO) event generators, such as MC@NLO [1] and POWHEG [2, 3], the answer is reasonably straightforward: use NLO PDF sets defined in a compatible factorization scheme. ${ }^{1}$ However, the number of processes implemented in a NLO Monte Carlo framework is still limited, and the use of LO Monte Carlo programs is more widespread. But for LO Monte Carlo event generators, the choice of the PDFs and their order is non-trivial.

In practice, most applications of LO event generators have been using available LO PDFs. Certain alternative practices, such as using NLO PDFs in LO event generators, have been also proposed to address some of the issues. It has been observed [5] that a better agreement with fully NLO predictions, both in terms of the shape and normalization of the cross section, and of acceptances calculated with experimental cuts, can often be obtained when LO event generators use NLO PDFs. But these alternatives have their own known drawbacks, particularly with the determination of the underlying event, and are not robust for all processes. ${ }^{2}$ The urgent need for better-performing event generator calculations has stimulated much discussion at recent conferences and workshops about PDFs that are tailor-made specifically for event generators. This general idea obviously makes sense; the question is how to construct these special PDFs?

To address this question, it is necessary to distinguish between two different sources of mismatches between the conventional LO PDFs and their event generator applications. The first problem is due to intrinsic limitations of the LO global analysis or LO calculations that make their "predictions" inherently unreliable at higher energies, beyond that of the input experimental data included in the global analysis (e.g., at the LHC), and for new physical processes that are not included in the global analysis (e.g., top quark and Higgs production). This problem has been discussed in literature [5]. Suggestions have been made [7] to remedy the known deficiencies of the LO calculations by relaxing constraints from the momentum sum rule and other common practices, basically by a trial-and-error approach determined by the a posteriori result. This paper will try to address this problem in a more direct way by utilizing the power of the global analysis itself and by going beyond its conventional method.

The second mismatch is associated with the initial-state radiation (ISR) that is present in the event generators, but not in the global analysis determining the LO PDFs. This problem, in principle, depends on the type of the event generator, given that each of them handles ISR differently. The differences in the ISR treatment should formally be taken into account when deriving the appropriate input PDF sets. In practice, at LO accuracy, the main impact of the radiation is kinematic in nature, with further subtleties being formally at NLO and thus beyond the scope of this study. In an initial-state parton shower, gluons are radiated at finite angles. In the DGLAP formalism used in global PDF fits, gluons

\footnotetext{
${ }^{1}$ This factorization scheme must agree with the specific algorithm for treatment of exclusive final states in the NLO event generator [4].

${ }^{2}$ The PYTHIA8 [6] framework allows one to use separate PDFs for the generation of the hard-scattering portion of the event and for the generation of the underlying event. Thus, one could use a NLO PDF for the matrix element and a LO PDF for the parton showering/underlying event. Formally, all parts of an event must be computed with the same PDF set; but, if the $x$ and $Q^{2}$ regimes where the two PDF sets are invoked are very different, the inconsistency should not be too serious.
} 
are assumed to be collinear. Thus, to produce, say, a $W$ or a Higgs boson at a particular rapidity, a larger momentum fraction for the incoming partons is required in a parton shower Monte Carlo program than in a fixed-order formalism, resulting in a kinematic suppression. We discuss the size of this suppression in section 3 and show that the effect, although noticeable, does not significantly affect predictions at the LHC, in comparison to more pronounced differences arising from the choice between the LO and NLO PDFs.

\section{Global analysis of PDFs for LO event generators}

Conventional global analyses determine PDFs by fitting theoretical QCD cross sections to the existing hard scattering data. Universality of PDFs and their calculable QCD evolution to higher scales allow us then to make predictions at higher energies and for new processes. For most applications, especially those relevant for event generators, this principle works well at NLO, since the accuracy of perturbative QCD predictions at this order usually matches the current and expected experimental precision. ${ }^{3}$ But when PDFs are determined in a LO global analysis, using existing experimental data, they are known to have incorrect behavior both at small and large partonic momentum fractions $x$, due to missing large terms that first arise in the hard matrix elements at a higher order (NLO). Many Tevatron/LHC cross sections tend to be larger at NLO than at LO for commonly used scales, i.e., the K-factor (the ratio of the NLO to LO cross sections) tends to be larger than 1 - see, for example, ref. [5] and table 4 later in this paper. As a consequence, when conventional LO PDFs are used in LO generators, predictions at high energies (such as at the LHC or, in some cases, the Tevatron) and for new physical processes can be quite unreliable, both in magnitude and shape [5, 7]. Alternative prescriptions, such as using LO matrix elements with NLO PDF sets, may better reproduce the shape of the full NLO cross section; but, as already mentioned, other issues with the normalization and underlying event still remain [5].

Since, by definition, we are constrained to use LO matrix elements in LO generators, this long-standing dilemma can be resolved - to the extent possible — only by trying to optimize factorization of LO cross sections, i.e., by finding better PDFs and possibly more sensible renormalization and factorization scales for each LO cross section. This can be carried out most systematically by redefining the goal and strategy of the global QCD analysis. ${ }^{4}$ In a conventional global analysis, the PDFs are optimized to fit the existing experimental data. For event generator applications, this is not the main purpose; rather, it is equally, if not more, important to produce reliable predictions at higher energies and for new processes. In fact, the efficacy of a PDF set for event generator applications, particularly LO ones, is mostly judged by how its predictions for future colliders meet expectations. ${ }^{5}$ But, prior to having real data at these colliders, what constitutes the

\footnotetext{
${ }^{3}$ For some processes, theoretical errors are exceptionally large; then even higher-order terms beyond NLO are needed.

${ }^{4}$ The need to rethink the strategy of the global analysis for event generators is also implicit in other attempts $[7,9]$ to address the same problem.

${ }^{5}$ And it is mainly on this ground that the conventional LO PDFs have been deemed unsatisfactory.
} 
correct expectations? This is where the existing NLO and NNLO calculations come in. There is a good reason to believe that, for standard model (SM) processes, the predictions of QCD at NLO and NNLO orders will be reasonably reliable. They can be used as a sensible substitute for nature (or "truth" as called in ref. [7]).

This observation immediately suggests that the most direct, and effective, way to obtain PDF sets for event generators is to generalize the conventional global QCD analysis to utilize the best estimates of key physical processes at future colliders (to ensure reliable predictions), in parallel with the existing experimental data sets (to ensure reasonable agreement with nature at currently available energy scales), as joint inputs to the global fitting. In principle, this idea can be applied at both LO and NLO; however it is only of practical interest for LO event generators at present. ${ }^{6}$ For this purpose, we can implement the constraints of "nature" at high energies in the form of pseudodata sets generated by NLO calculations for representative physical processes that are sensitive to various flavors of partons: light quarks, gluons, and heavy quarks.

Even if this basic idea of a global analysis of PDFs optimized for event generators is quite simple and natural, a few relevant considerations need to be pointed out before going into details. First, since we are focusing on PDFs for LO generators, we must use LO matrix elements in the calculations for the global fitting. But we know already that LO matrix elements provide only the most basic approximations to the true theory; therefore, even the most optimized PDFs cannot be expected to fit well both the lower-energy experimental data, especially in deep-inelastic scattering processes, and the higher energy pseudodata at the same time. These PDFs represent the best compromise that can be obtained within the restrictions of the LO matrix element approximation. They are intended solely as an input to LO event generators for predictions at the $L H C$, with an eye on their inherent limitations.

In its typical application, a LO event generator is not used to predict absolute cross sections per se, but rather to calculate detector acceptances for, and backgrounds to, physics processes of interest, in conjunction with detailed detector simulations. It is desirable that the LO event generators produce reasonably accurate normalizations for the cross sections, although it is understood that higher-order contributions not included in the LO generators may introduce sizeable corrections. But it is even more important that kinematic shapes, such as rapidity distributions, be accurately described, so that event acceptance derived from these distributions is close to reality.

It should be readily emphasized that the PDFs generated in this modified manner are not "leading-order PDFs" - rather, they are "PDFs for leading-order Monte-Carlo programs", or "LO-MC PDFs". This distinction needs to be made, since there are still lingering misconceptions about the need to "match orders" in literature and in public discussions. Event generators, including "LO event generators", have some elements of higher-order contributions and, in this sense, are not at the stated order in the QCD coupling. Because of these two considerations, the global fits we are performing have the freedom of choice on several fronts, all of which can impact the numerical results.

\footnotetext{
${ }^{6}$ To improve NLO PDFs for use with NLO event generators, one could supplement existing experimental data input by predicted high energy cross sections calculated in NNLO, whenever these calculations are available.
} 
The order of $\alpha_{s}$ : We have the choice of using either a 1-loop or 2-loop version of the QCD coupling $\alpha_{s}$. Nominally, a 1-loop $\alpha_{s}$ may be considered as more appropriate with a LO event generator, but some parton showering models prefer a 2-loop $\alpha_{s}$. We also have the freedom to set $\alpha_{s}$ free in the global fit or to tie it to the world average. We choose to fix $\alpha_{s}\left(M_{Z}\right)$ at the world average (0.118 at two loops and 0.130 at one loop), for convenience and compatibility with the previous CTEQ PDF sets.

Factorization scales: should the renormalization and factorization scales for different processes be fixed, or could they be discretionally chosen, or even be fitted, in order to get the best agreement? The motivation for considering flexibility here is because LO calculations are notoriously scale-dependent for most processes. Changes in the scales can affect both the normalization and the shapes of the Tevatron and LHC cross sections. But this flexibility can be also employed to advantage, by finding the LO scale values that provide the best approximation to the real data and NLO pseudodata. ${ }^{7}$

Momentum sum rule: this sum rule relates PDFs or different flavors in order to conserve the total momentum carried by the partons. Can it be relaxed in this kind of global analysis? This possibility was brought forth by earlier attempts to fix the problems of existing LO PDFs [7] by putting more gluons in the high- $x$ region than otherwise would be allowed in a LO fit. Is this still needed in our approach, which automatically puts more partons into the relevant $x$ region because of the constraints imposed by the NLO pseudodata? Even when relaxation of the momentum sum rule is not required, could it still improve the results? We shall answer these questions by performing parallel global analyses with and without enforcing the momentum sum rule and by comparing their outcomes.

Selection and construction of NLO pseudodata sets to represent "nature" at high energies: there is clearly a great deal of latitude in doing this. The selection of physical cross sections to be represented in these theoretical data sets is guided by the importance of the process for the LHC physics, and by the parton flavors that these processes are sensitive to. One would like to ensure that all parton flavors, in most ranges of $x$, are covered by these constraints. Since these pseudodata sets are used in a fitting procedure, one must also assign "errors" to each data point, as well as overall weights of the $\chi^{2}$ values contributed by each pseudodata set. These can be guided by the estimated theoretical and expected experimental errors, but are ultimately subjective.

To satisfy phenomenological considerations, the LO-MC PDFs should

- behave similarly to the usual LO PDFs as $x \rightarrow 0$ (assumed by the current models for the underlying event) and to NLO PDFs as $x \rightarrow 1$;

- describe the underlying event at the Tevatron (with a Monte-Carlo tune similar to what is currently used) and extrapolate to a reasonable level of underlying event at the LHC.

\footnotetext{
${ }^{7}$ The scale dependence is usually moderated at NLO. In our global fits at NLO, the scales are always fixed at their nominal values.
} 
Th NLO pseudodata for the LHC scattering processes included in the fit is chosen so as to enforce this desired behavior of the LO-MC PDFs. As such, we use the singleinclusive $W^{+}, W^{-}$and $Z^{0}$ rapidity distributions (affecting the low- $x$ and high- $x$ quark distributions), the $b \bar{b}^{8}$ and $t \bar{t}$ invariant mass distributions, and the rapidity distribution for a $120 \mathrm{GeV}$ standard model Higgs boson produced through $g g$ fusion (affecting the low- $x$ and high- $x$ gluon distribution). All NLO pseudodata cross sections were computed using the MCFM program [11] and CTEQ6.6M PDFs [12].

When generating the vector boson and the Higgs boson NLO pseudodata, we have set the renormalization/factorization scale to be equal to the (pole) mass of the respective boson. For the scale in $t \bar{t}$ production, we have used the top quark mass $(172 \mathrm{GeV})$, and, for the scale in $b \bar{b}$ production, we have used the invariant mass of the quark-antiquark pair. All pseudodata cross sections were computed at $14 \mathrm{TeV}$, the nominal center-of-mass energy of the LHC. After the fit, we also checked the level of agreement between the NLO predictions and the $\mathrm{LO}-\mathrm{MC}$ predictions at $7 \mathrm{TeV}$ and $10 \mathrm{TeV}$, the initial running energies of the LHC.

To illustrate the scale of the problem we are trying to address, figure 1 shows rapidity distributions for inclusive $W^{ \pm}, Z^{0}$, and Higgs boson production at $\sqrt{s}=14 \mathrm{TeV}$, the key LHC processes. They are computed by the MCFM program at NLO using the CTEQ6.6M NLO PDFs, and at LO using the LO CTEQ6L1 [13] and NLO CTEQ6.6M PDFs, with the same scale choices.

As expected, the average normalization of the cross section with the LO hard part is smaller than that at NLO, regardless of whether the LO PDFs or NLO PDFs are used. In addition, comparison of the "LO-LO" CTEQ6L1 and "LO-NLO" CTEQ6.6M distributions reveals significant differences in the shapes, obviously caused by the input PDFs and not by the different orders of the hard matrix elements. While such differences are observed in all four processes, the $W^{+}$rapidity distribution provides a particularly eye-catching example of the danger of using the conventional LO PDFs with an LO hard cross section (or LO event generators). The strong forward-backward peaking of the "LO-LO" CTEQ6L1 $W^{+}$ rapidity distribution disappears when the NLO CTEQ6.6M PDFs are used with the LO hard part. ${ }^{9}$ The acceptance for $W^{+} \rightarrow e^{+} \nu$, computed by a LO event generator for standard analysis cuts, differs when the NLO CTEQ6.6 PDFs are used instead of CTEQ6L1. It is thus a misconception that the strong forward-backward peaking observed in the prediction based on CTEQ6L1 is a benchmark feature of inclusive $W^{+}$rapidity distribution at the LHC. In reality, it is primarily an artifact due to inadequacies of the conventional LO fitting formalism.

The disagreements with the NLO benchmark cross sections are greatly reduced when

\footnotetext{
${ }^{8}$ Here, we consider $b \bar{b}$ production only through $g g$ fusion (with the $b$ quark mass set equal to $4.75 \mathrm{GeV}$ ), to constrain the gluon PDF in the low- $x$ range typical for the underlying event in hard scattering collisions. We fit the $b \bar{b}$ mass range from $10-100 \mathrm{GeV} / c^{2}$. Hereafter, we refer to this process as $b^{\prime} \overline{b^{\prime}}$ since it refers to a restricted set of production subprocesses.

${ }^{9}$ This peaking is caused by the increased magnitude of the CTEQ6L1 $u$-quark distribution at large $x$, as compared to its CTEQ6.6M counterpart. The same large- $x$ enhancement of the LO $u$ quarks leads to anomalously large predictions for ultra-heavy $t \bar{t}$ pair production at the Tevatron.
} 

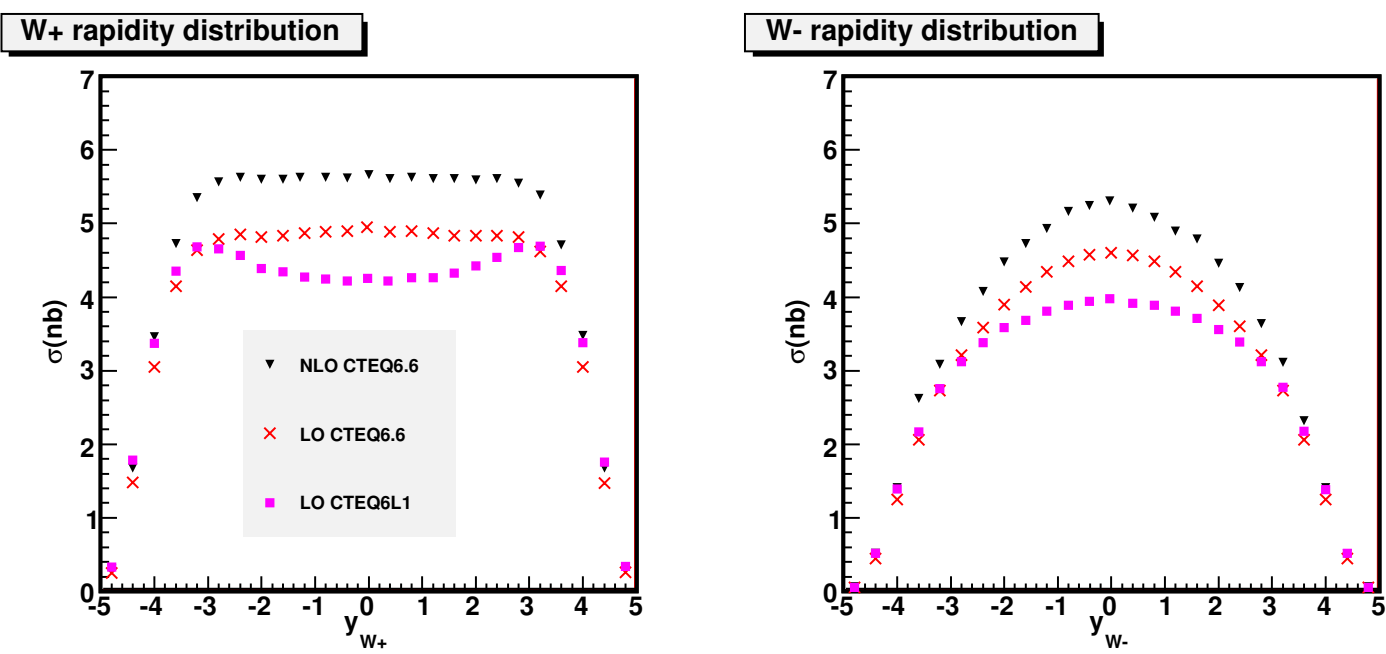

\section{Z rapidity distribution}
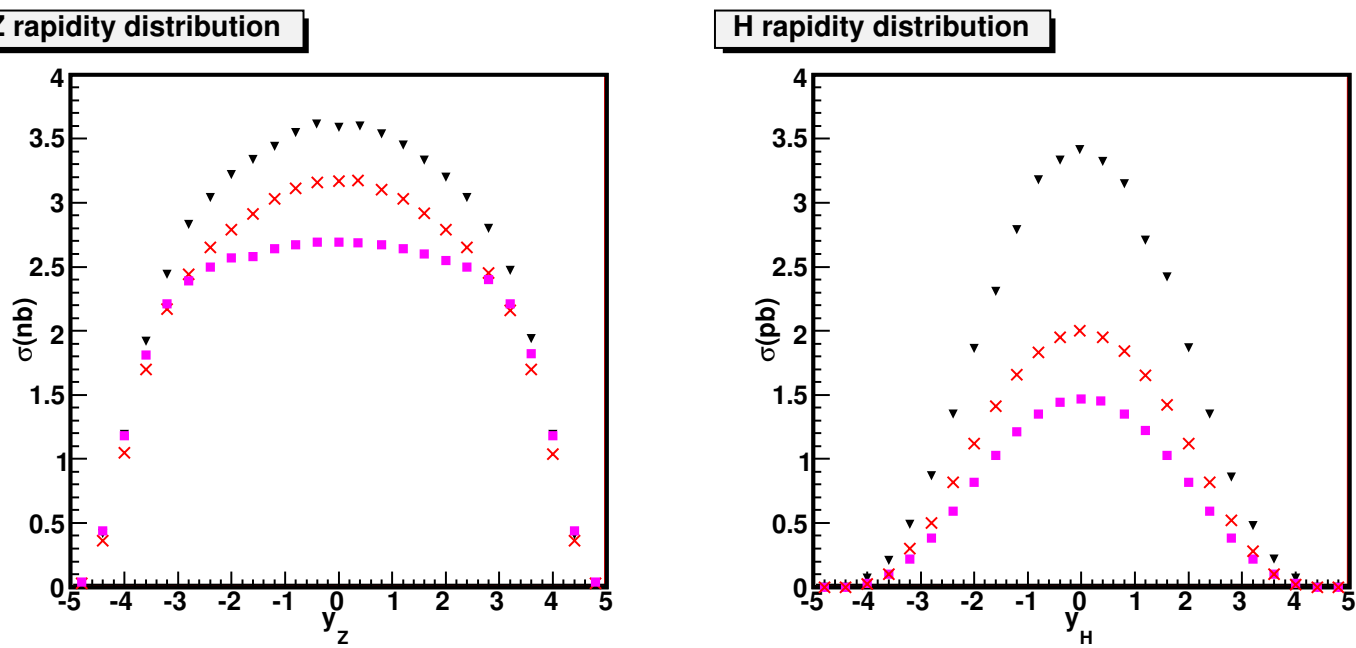

Figure 1. A comparison of the NLO pseudodata for SM boson rapidity distributions (in $\Delta y=0.4$ bins) predicted at the $\mathrm{LHC}(14 \mathrm{TeV})$ to the respective LO predictions based on CTEQ6.6M and CTEQ6L1 PDFs.

the LO cross sections are computed using our LO-MC PDFs, as will be shown in section 4.2. It is also obvious, from the above description, that there is a wide range of possible ways to implement our general approach.

\section{$3 \quad$ Impact of parton showering}

The LO-MC PDFs in our study are constructed using fixed-order (sometimes called "parton-level") QCD calculations. In practice, these PDFs will be used with LO matrix elements embedded into a parton shower framework. According to initial-state radiation algorithms, shower partons are emitted at non-zero angles with finite transverse momentum, and not with a zero $k_{T}$ implicit in the collinear approximation. It might be argued that the resulting kinematic suppression due to parton showering (handled differently by 
various event generators) should be taken into account when deriving PDFs for explicit use in Monte Carlo programs. ${ }^{10}$

To quantify kinematical dependence of this suppression, figure 2 examines several leading-order rapidity $(y)$ distributions for SM Higgs boson production via $g g$ fusion, obtained in the PYTHIA event generator [6]. We compare cross sections with and without initial-state radiation (ISR) contributions, for either the CTEQ6L1 PDFs or one of our new LO-MC PDF sets CT09MC2 (to be described later). In the top left figure, distributions for a (toy) $10 \mathrm{GeV}$ mass Higgs boson at the LHC energy $\sqrt{s}=10 \mathrm{TeV}$ are considered. A sizeable kinematic suppression in the presence of ISR is evident at forward rapidities, while the total cross section (integrated over the whole rapidity range) remains largely unaffected. These features force the rapidity distribution of such an ultra-light Higgs boson to be more central with the initial-state parton showering on than without it.

In production of a heavier Higgs boson with mass $120 \mathrm{GeV}$ (top right figure), the effects of the kinematic suppression at forward rapidities are still evident, but reduced in magnitude. For Higgs boson with mass $300 \mathrm{GeV}$ (bottom figure), the effects of the kinematic suppression are reduced still further. This behavior indicates that parton showering is not likely to affect greatly the rapidity distributions for large-mass phenomena at the LHC, such as for example, $t \bar{t}$ production.

A comparison of PYTHIA predictions for production of a $W^{+}$boson at the LHC $(10 \mathrm{TeV})$ with and without parton showering is shown in figure 3. For both CTEQ6L1 and CT09MC2 PDFs, alterations in the shape of the rapidity distribution caused by the parton showering are relatively small. In particular, it can be noted that the differences in the shape of $W^{+}$rapidity distributions introduced by conventional LO PDFs (such as CTEQ6L1), as compared to the NLO cross section, are largely unaffected by the parton showering. The choice of the PDF set evidently outweighs the impact of parton showering in the case of $W$ boson production.

In general, the use of the LO-MC PDFs shifts the production of gauge bosons to more central values of rapidity. A similar shift occurs because of parton showering, but the magnitude of the shift decreases as the mass of the final state increases. The impact of the showering also decreases for higher center-of-mass energies $(14 \mathrm{TeV}$, for example, as compared to $10 \mathrm{TeV}$ ). For the rest of the paper, unless noted, we will use fixed-order predictions, although extensive comparisons have been made with parton-shower predictions as well.

\section{Results of the modified PDF analysis}

\subsection{General considerations}

The LO-MC PDFs (designated as CT09MC PDFs) are constrained by including the same existing experimental data sets as those used in the CTEQ6.6 PDF analysis [12], combined with the pseudodata on NLO cross sections for five representative LHC scattering processes

\footnotetext{
${ }^{10}$ This was first pointed out to us by Hannes Jung.
} 

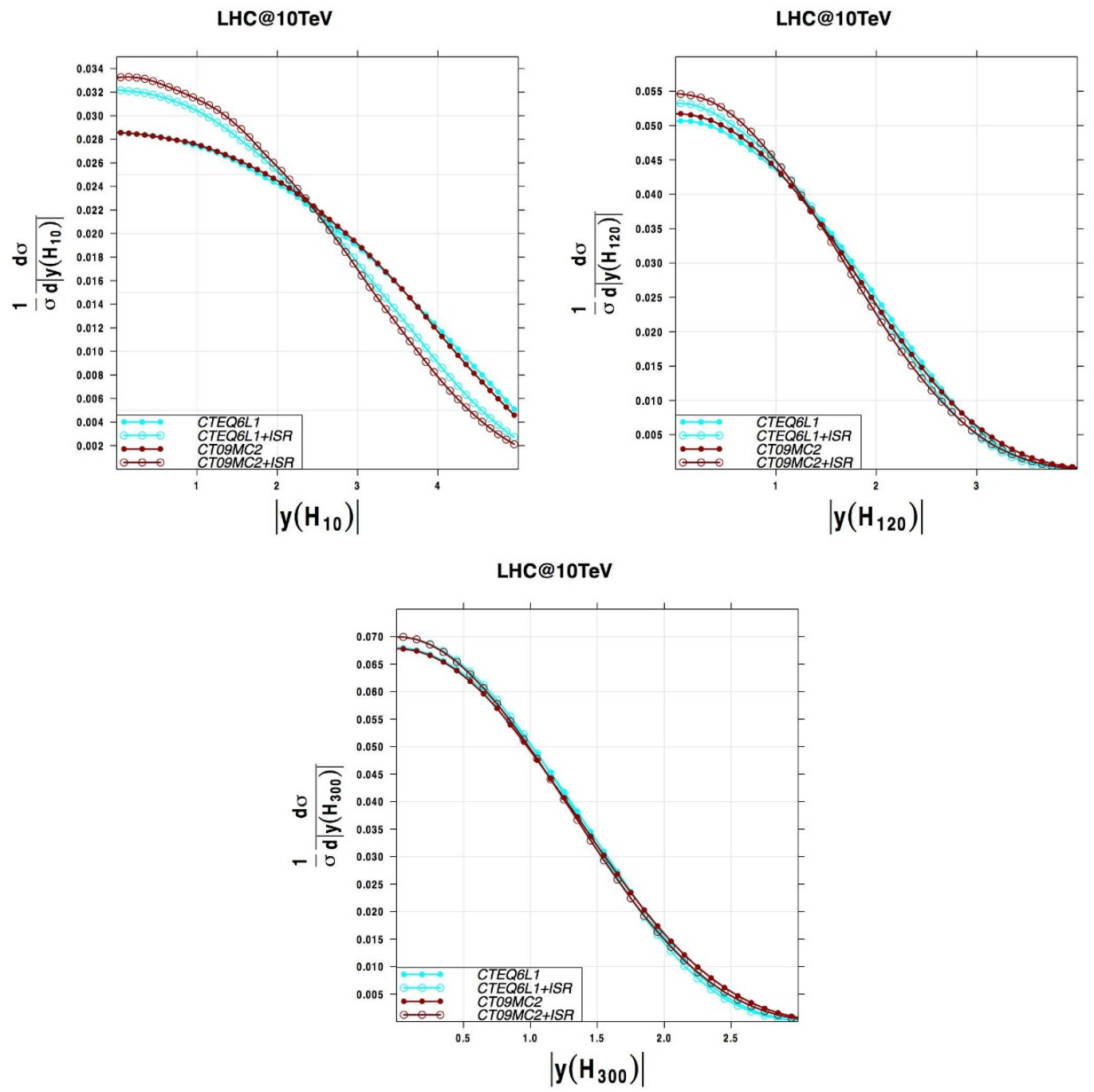

Figure 2. PYTHIA predictions for production of a $10 \mathrm{GeV}$ Higgs boson (top left), a $120 \mathrm{GeV}$ Higgs boson (top right), and a $300 \mathrm{GeV}$ Higgs boson (bottom) via the $g g \rightarrow H$ process at the LHC (at $\sqrt{s}=10 \mathrm{TeV}$ ), with and without contributions from the initial-state radiation. Distributions in the absolute value of the Higgs boson's rapidity $|y|$ are shown.

discussed in section 2. Correlated systematic error information is used for all experimental data sets.

To give an idea about the impact the NLO radiative contributions, a fully NLO global fit in the CTEQ framework, with no pseudodata, results in a $\chi^{2} /$ d.o.f. close to 1 for a sample of around 2700 data points. If the fit is carried out instead at LO, with a 1-loop $\alpha_{s}$, the $\chi^{2}$ worsens by about $30 \%$. If $\alpha_{s}$ is evaluated at two loops, the $\chi^{2}$ is larger than that at NLO by $20 \%$; i.e., the 2-loop $\alpha_{s}$ experssion improves $\chi^{2}$ in the LO fit by about $10 \%$. Thus, the data prefer more rapid variation of $\alpha_{s}$ with $Q^{2}$ provided by its two-loop expression.

If, in addition, the momentum sum rule is relaxed, modest improvements in the global $\chi^{2}$ are observed, accompanied by a violation of the momentum fraction sum on the order of $3 \%$. Allowing more gluon momentum does improve the LO-MC fit to some of the (regular) 


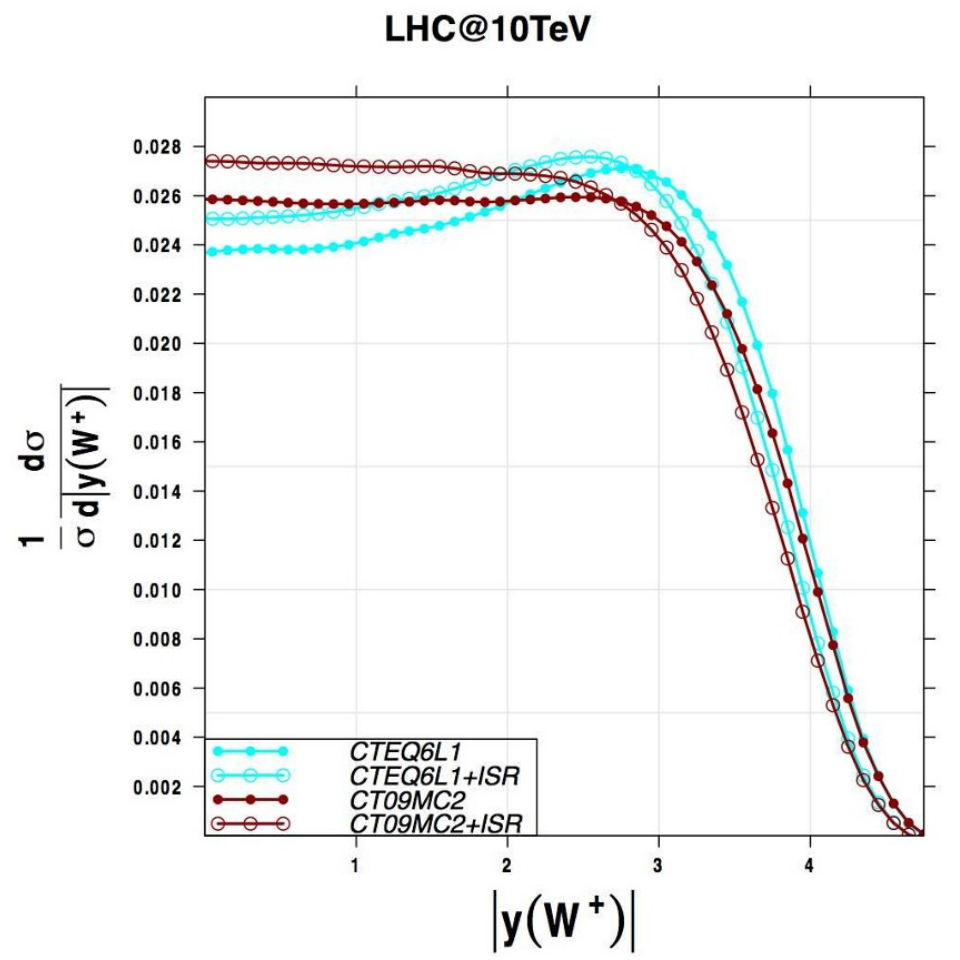

Figure 3. PYTHIA predictions for rapidity distributions of a $W^{+}$boson produced via $q^{\prime} \bar{q} \rightarrow W^{+}$ process at the LHC (at $\sqrt{s}=10 \mathrm{TeV}$ ), computed with CTEQ6L1 PDFs and CT09MC2 PDFs, with and without contributions from the initial-state radiation.

data sets, but results in a worse fit to other data sets. Thus, we find it difficult to achieve as small a value of $\chi^{2}$ in the LO-MC fits as in the NLO fit, even when the momentum sum rule is relaxed.

\section{$4.2 \quad$ Numerical results}

\subsubsection{CT09MCS PDFs}

We now consider the LO-MC PDFs produced with the NLO pseudodata included in our data set. First, we consider the case where the momentum sum rule is kept intact, but the factorization scales in the LO matrix elements corresponding to the pseudodata are allowed to vary as free parameters. The normalization of the LO calculation for each pseudodata set $i$ is also allowed to float to reach the best agreement with the NLO cross section, which is equivalently described by a floating normalization of each pseudodata set, denoted by $N_{i}$. The effective K-factor (NLO/LO) for the pseudodata is then given by $K_{i}=1 / N_{i}$. We will name the LO-MC PDF set resulting from this approach as "CT09MCS", where $S$ signifies the varied scales in the fit to the pseudodata.

In practical terms, the factorization scale $\mu_{i}$ for each pseudodata set, taken to be the same as the renormalization scale, is allowed to vary within a factor of four around the nominal scale defined for each process in section 2. A $\chi^{2}$ penalty is assigned for deviations of the normalization $N_{i}$ from unity, and the weights applied to $\chi^{2}$ values from the pseudodata 


\begin{tabular}{|c|c|c|c|c|c|c|}
\hline & $W^{+}$ & $W^{-}$ & $Z$ & $H$ & $t \bar{t}$ & $b^{\prime} \overline{b^{\prime}}$ \\
\hline$\mu_{i}$ & $1.96 M_{W}$ & $1.96 M_{W}$ & $1.96 M_{Z}$ & $1.06 M_{H}$ & $1.41 M_{t}$ & $0.40 M_{b^{\prime} \overline{b^{\prime}}}$ \\
\hline$K_{i}$ & 1.11 & 1.09 & 1.09 & 1.87 & 2.09 & 4.09 \\
\hline
\end{tabular}

Table 1. The fitted $\mu_{i}$ and $K_{i}$ for each pseudodata set, obtained using CT09MCS PDFs. As a reminder, $b^{\prime} \overline{b^{\prime}}$ refers to production only through the $g g$ sub-process.
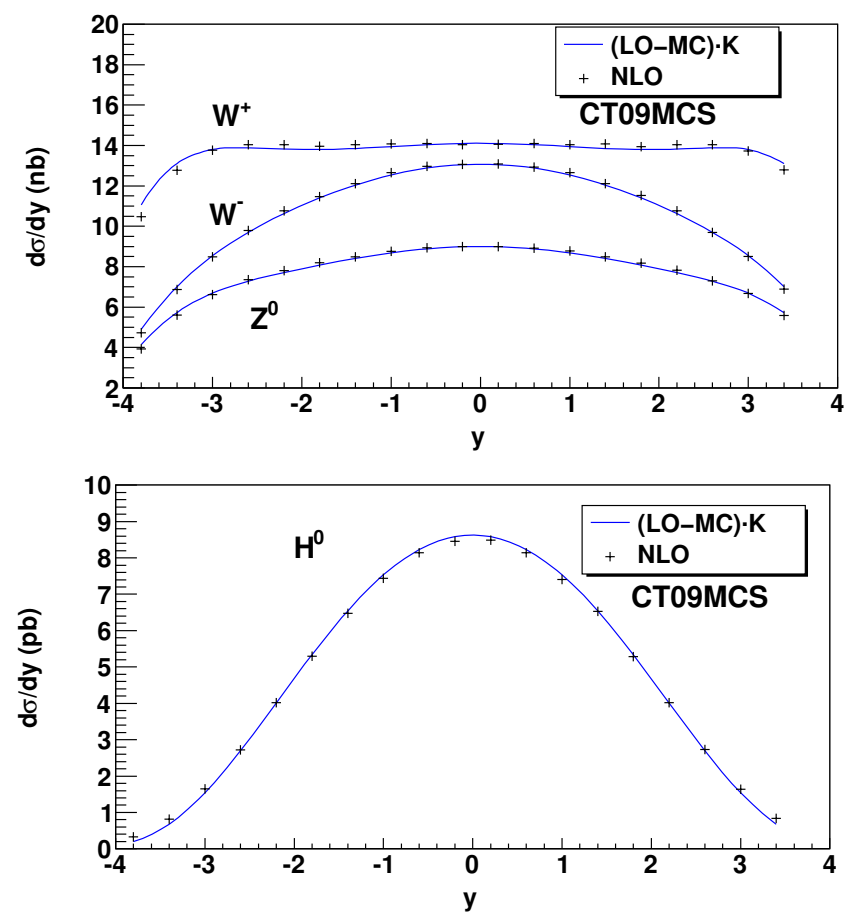

Figure 4. Comparison of the NLO pseudodata cross sections for $W, Z$ and Higgs production at the LHC (14 TeV) with the LO predictions using CT09MCS PDFs. The scale choices and effective $\mathrm{K}$-factors applied to the LO-MC cross sections are listed in table 1.

sets can be varied as well. For this exercise, we use only the 2-loop $\alpha_{s}\left(m_{Z}\right)$. As stated previously, the value of the (2-loop) $\alpha_{s}\left(m_{Z}\right)$ is fixed at the value of 0.118 used in the CTEQ6.6 global fit.

In the CT09MCS approach, the optimum $\chi^{2}$ is obtained with the scales given in table 1, with each scale being within a factor of 2 or so from the nominal value. A comparison of the CT09MCS predictions with the NLO pseudodata is presented in figures 4 and 5. The NLO cross sections are shown with their true normalization, while the LO-MC cross sections are multiplied by the best-fit K-factors listed in table 1. Excellent agreement between the CT09MCS and NLO cross sections is observed for all four scattering processes.

\subsubsection{CT09MC1 and CT09MC2 PDFs}

In the second approach, we again fit the real experimental data and NLO pseudodata together, but relax the momentum sum rule and fix the factorization scales at their nominal 

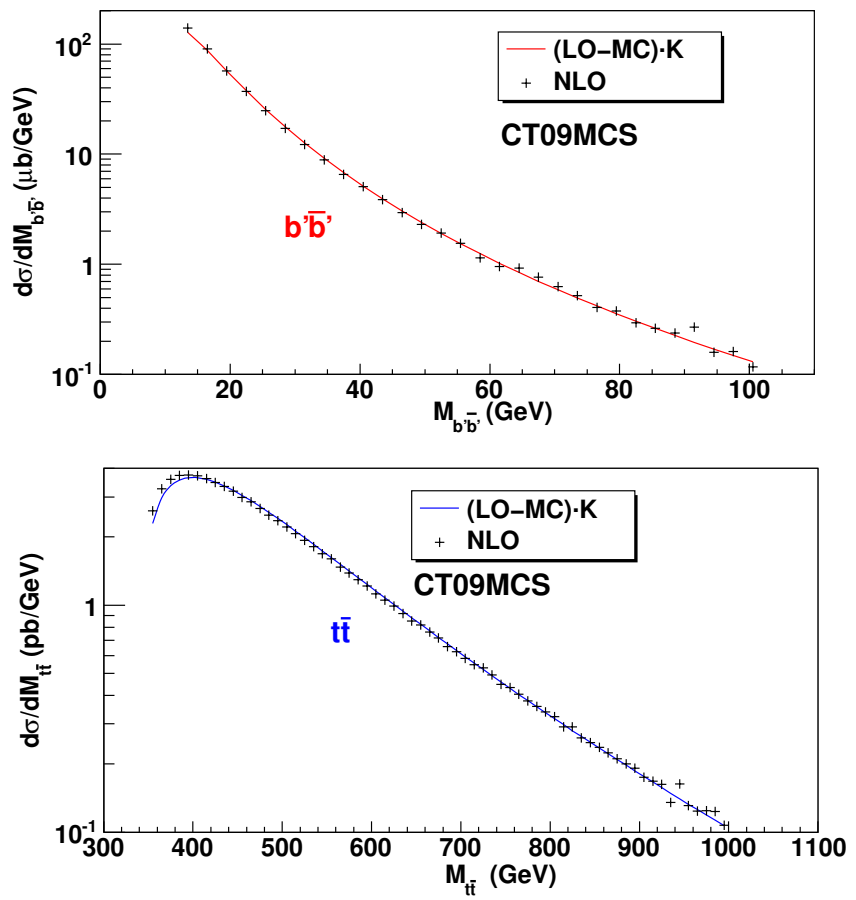

Figure 5. Comparison of the NLO pseudodata cross sections for $b^{\prime} \overline{b^{\prime}}$ and $t \bar{t}$ production at the LHC $(14 \mathrm{TeV})$ with the LO predictions using CT09MCS PDFs. The scale choices and effective K-factors applied to the LO-MC cross sections are listed in table 1.

values. The pseudo-data normalizations are allowed to float, as before. We obtain two PDF sets, designated as CT09MC1 and CT09MC2, determined with the 1-loop and 2loop expressions for $\alpha_{s}$, respectively. In this approach, good agreement with the NLO pseudodata is reached only at the expense of a worse agreement with the real data. We balance between describing the real data and LHC pseudodata by assigning an extra weight to the pseudodata to better reproduce the pseudodata's normalization and shape. As the weight of the pseudodata in the global fit is increased, (i) the pseudodata normalizations get closer to unity, (ii) larger violation of the momentum sum rule is observed, (iii) the quality of agreement with the real data sets deteriorates progressively, with $\chi^{2}$ values for the real data being worse by 10-20\% for the CT09MC1 and CT09MC2 fits than without the pseudodata. The 2-loop $\alpha_{s}$ expression results in slightly lower normalizations $N_{i}$ for the pseudodata sets and a slightly larger violation of the momentum sum rule than in the case of the 1-loop $\alpha_{s}$, but in a similar level of agreement with the real data set.

The final CT09MC1 and CT09MC2 PDFs thus present a compromise that tries for a better shape and normalization for the pseudodata without sacrificing reasonable (LO) description of the real (non-LHC) data sets.

\subsubsection{CT09MC2 predictions for selected LHC cross sections}

Comparison of CT09MC2 predictions to the NLO pseudodata at the LHC center-of-mass energies $\sqrt{s}=14,10$, and (for some processes) $7 \mathrm{TeV}$ is shown in figures $6-11$. Similar 


\begin{tabular}{|c|c|c|c|c|c|c|c|}
\hline & $W^{+}$ & $W^{-}$ & $Z$ & $H$ & $t \bar{t}$ & $b^{\prime} \bar{b}^{\prime}$ & momentum sum \\
\hline$K_{i}$ (CT09MC1) & 1.00 & 0.99 & 0.98 & 1.22 & 1.09 & 2.70 & 1.10 \\
\hline$K_{i}$ (CT09MC2) & 1.02 & 1.00 & 1.00 & 1.32 & 1.09 & 3.13 & 1.14 \\
\hline
\end{tabular}

Table 2. Fitted $K_{i}$ for each pseudodata set at the LHC (at $14 \mathrm{TeV}$ ) for CT09MC1 and CT09MC2 PDFs, along with the sum of parton momentum fractions in the proton for each set.

values of cross sections are obtained with the CT09MC1 PDF set. In the figures, the actual cross sections are compared, without applying any normalization factors.

In all cases, the LO cross sections based on the CT09MC2 PDFs are closer to the NLO predictions both in the overall normalization and shape than the respective LO cross sections based on a standard LO PDF such as CTEQ6L1. The predictions for $W$ and $Z$ production at LO-MC are almost identical to those at NLO, and those for $t \bar{t}$ production are considerably closer to the NLO predictions. ${ }^{11}$ The predictions for the production of a $120 \mathrm{GeV}$ Higgs boson are similar in shape, but the LO-MC prediction is still significantly lower than NLO (see the discussion below). The LO-MC predictions have a similar or even better agreement with the NLO benchmark cross sections at $\sqrt{s}=7$ and $10 \mathrm{TeV}$ than at $14 \mathrm{TeV}$.

An alternative set of PDFs (MRST2007lomod) for leading-order Monte Carlo programs was developed in ref. [7]. The figures compare the LO predictions utilising the MRST2007lomod PDFs with our results. At $7 \mathrm{TeV}$, the difference between the LO predictions for $W$ and $Z$ production using the MRST2007lomod PDFs and the NLO benchmark cross sections is essentially a normalization shift. At $10 \mathrm{TeV}$, and then especially at $14 \mathrm{TeV}$, there is also a noticeable difference in the shape of the rapidity distribution. However, both CT09MC2 and MRST2007lomod predictions provide an almost identical description for Higgs production.

The K-factors that need to be applied to the LO CT09MC1 and CT09MC2 predictions to recoincile them with their NLO counterparts are listed in table 2. The K-factors for $W$ and $Z$ boson production are basically unity, made possible by the extra freedom introduced by the relaxation of the momentum sum rule. The K-factors for the gluon-induced processes are closer to unity than for the standard LO PDF, as a result of the larger gluon density at high $x$. The K-factor for Higgs production remains significantly larger than unity, since the virtual corrections to this process are especially large and cannot (nor should they) be completely compensated by an increase in the LO gluon density.

\section{Comparisons of PDFs}

Figures 12-15 compare the LO-MC PDFs described in this paper with the CTEQ6.6M and CTEQ6L PDFs, for various parton flavors and energy scales. The LO-MC gluon

\footnotetext{
${ }^{11}$ Both the LO and NLO predictions for $t \bar{t}$ production are evaluated at the factorization scale $\mu=m_{t}$. The impact of using a different scale $\mu=\sqrt{\hat{s}}$ is also shown in figure 11, indicating the large scale dependence present in LO predictions.
} 


\section{W+ rapidity distribution}

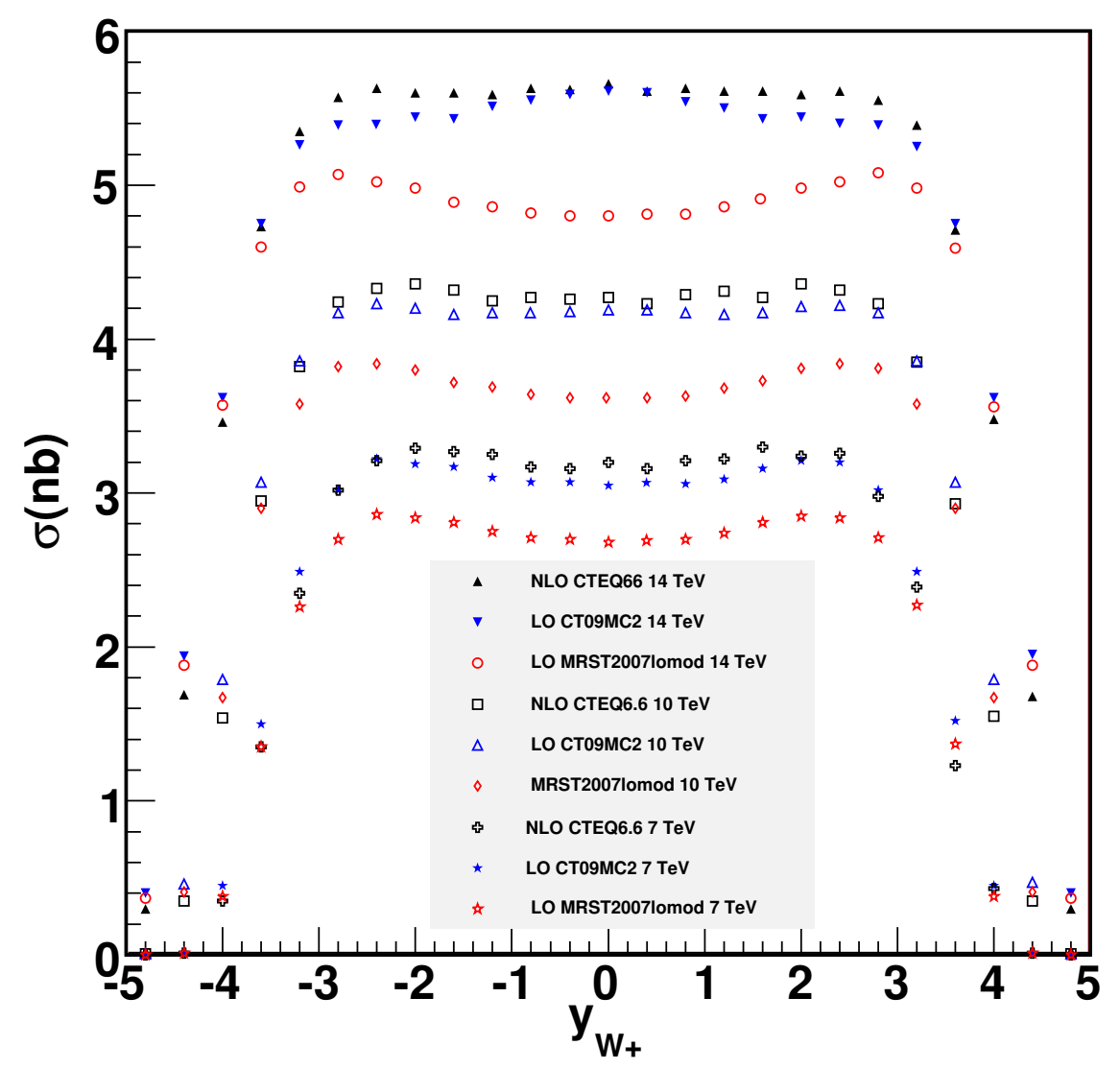

Figure 6. Predictions for the $W^{+}$rapidity distribution at the $\operatorname{LHC}(\sqrt{s}=7,10$ and $14 \mathrm{TeV})$ in $\Delta y=0.4$ bins, given at NLO using the CTEQ6.6M PDFs, and at LO using the CT09MC2 and MRST2007lomod PDFs. The actual cross sections (without normalization rescaling factors) are shown.

PDF CT09MCS, obtained with the fitted normalizations and scales, is quite close to the conventional LO PDF, CTEQ6L, as seen in figure 12. The gluon distributions in two LOMC fits with the relaxed momentum sum rule, CT09MC1 and CT09MC2, are equal to, or larger than, CTEQ6L in the entire $x$ range. They are larger than the CTEQ6.6M gluon up to $x$ values of 0.1 (CT09MC1) and 0.4 (CT09MC2). All LO-MC gluon PDFs approach the CTEQ6L gluon PDF at small $x$ (0.001 or less), in the region responsible for producing the underlying event at the LHC. With the momentum sum rule relaxed, the 2-loop CT09MC2 gluon is noticeably larger than the 1-loop CT09MC1 gluon, in order to compensate for the smaller value of the 2-loop QCD coupling strength when fitting the NLO pseudodata. ${ }^{12}$

The increase in the CT09MC1 and CT09MC2 gluon distributions is accompanied by the significant increase in the small- $x$ sea quark distributions. The LO-MC $u$-quark dis-

\footnotetext{
${ }^{12}$ Such increase does not happen if the momentum sum rule is enforced. For example, the CTEQ6L1 gluon PDF (with 1-loop $\alpha_{s}$ ) is about the same as the CTEQ6L gluon PDF (with 2-loop $\alpha_{s}$ ).
} 


\section{W- rapidity distribution}

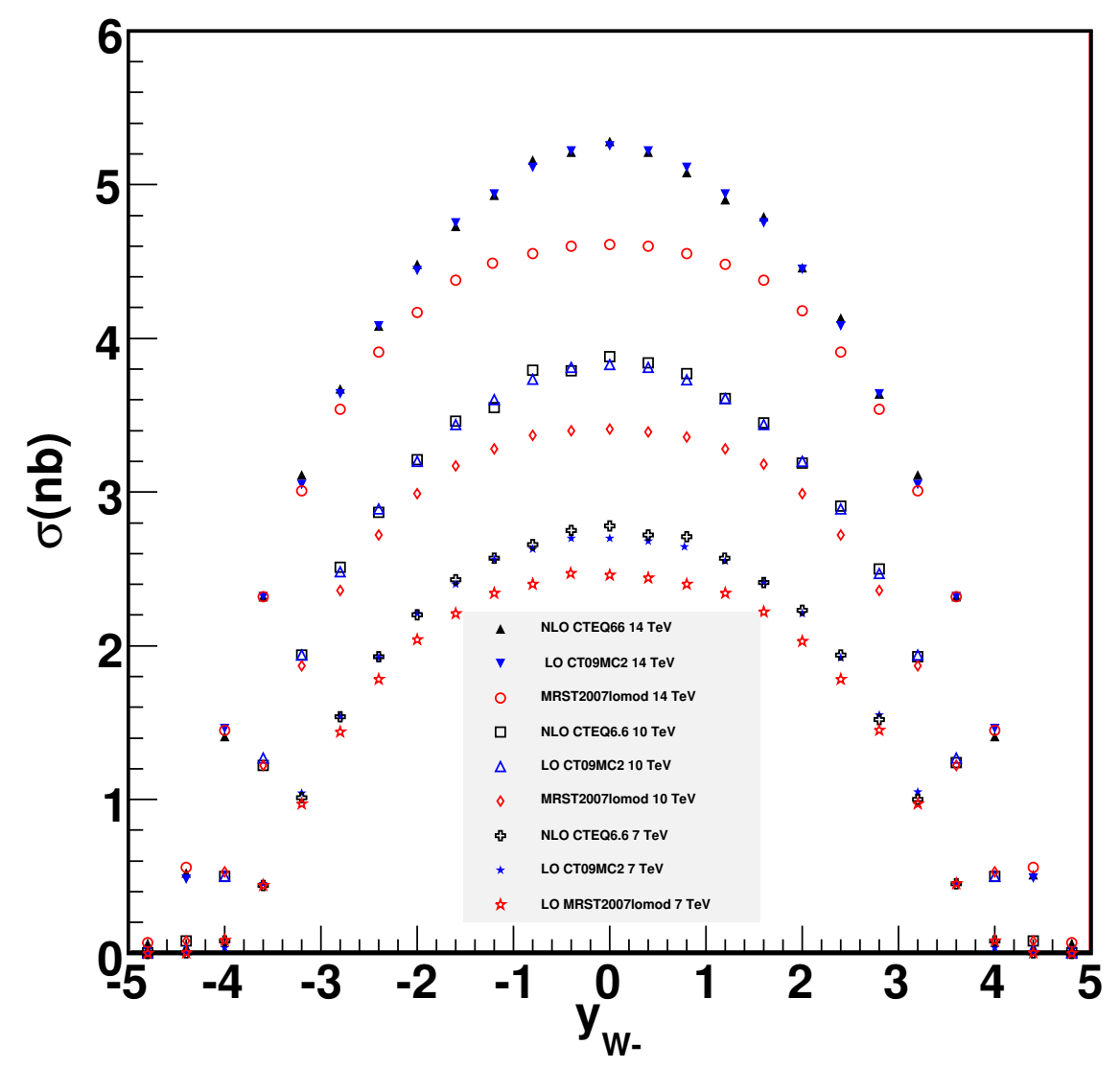

Figure 7. Same as figure 6, for the $W^{-}$rapidity distribution.

tributions (cf. figure 13) remain larger than the NLO $u$-quark distribution at $x>0.2$, in a manner similar to the conventional CTEQ6L $u$-quark distribution. The $u, \bar{u}, d$, and $\bar{d}$ distributions are larger than CTEQ6.6M and CTEQ6L at small and moderate $x$, leading to both a flattening of the LHC $W^{+}$rapidity distribution and an increase in the total cross sections for the vector boson pseudodata required by the full NLO calculations. Finally, while the CT09MC PDFs for (anti)quarks are quite different from their MRST2007lomod counterparts, the CT09MC gluon distributions are similar to those from MRST except at high $x$, where the CT09MC PDFs are closer to CTEQ6.6M due to the influence of the pseudodata.

\section{Predictions for other LHC cross sections}

By construction, predictions based on the LO-MC PDFs provide a better description of the LHC pseudodata cross sections. The pseudodata sets were chosen so as to be representative of the universally desired PDF behavior for typical LHC hard-scattering cross sections, but it is important to check predictions for the cross sections that were not a part of the 


\section{$Z$ rapidity distribution}

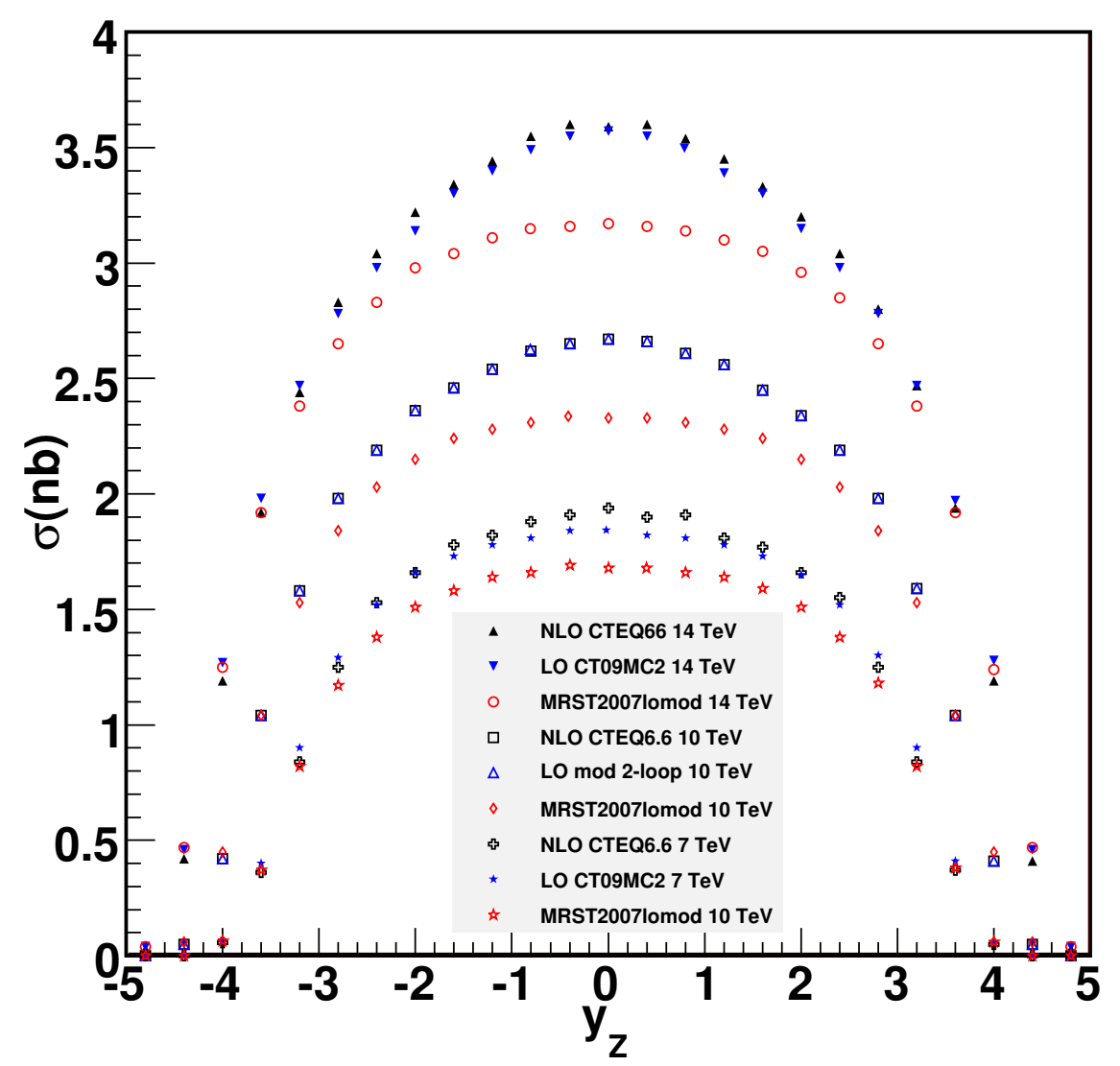

Figure 8. Same as figure 6 , for the $Z$ rapidity distribution.

pseudodata sets. In figure 17, we show cross sections for vector boson fusion production of a SM Higgs boson $\left(m_{\text {Higgs }}=120 \mathrm{GeV}\right)$, computed at NLO using the CTEQ6.6M PDFs, and at LO using two LO-MC PDFs (CT09MCS and CT09MC2). Distributions in the rapidities of the Higgs boson and the leading jet are plotted. The two LO-MC calculations reproduce well the shapes of the NLO rapidity distributions. The LO-MC cross sections are larger (smaller) than the respective NLO cross sections when the CT09MC2 (CT09MCS) PDFs are used. Both of them differ from the NLO (CTEQ6.6M) prediction in the central rapidity region by about ten percent.

To study the impact of the LO-MC PDFs on the matching of (multi-parton) hard matrix elements with parton showers, we have performed a comparison of parton-level cross sections for production of $W^{+}+\mathrm{n}$-partons $(\mathrm{n}=0, \ldots, 4)$ at the LHC $(10 \mathrm{TeV})$, computed by the MADGRAPH program [14] with both the conventional (CTEQ6L1) and CT09MC2 PDFs. We have found that the CT09MC2 PDFs increase the subprocess cross sections by a factor of about 1.25-1.35 for $q \bar{q}$ and $g q$ initial states, relatively independently of the flavors of the initial-state quarks and the number of partons in the final state. For $g g$ initial states, the factor is larger, ranging from about 1.5 to 1.75 . The details of this comparison are collected in appendix A. 


\section{SM Higgs boson rapidity distribution}

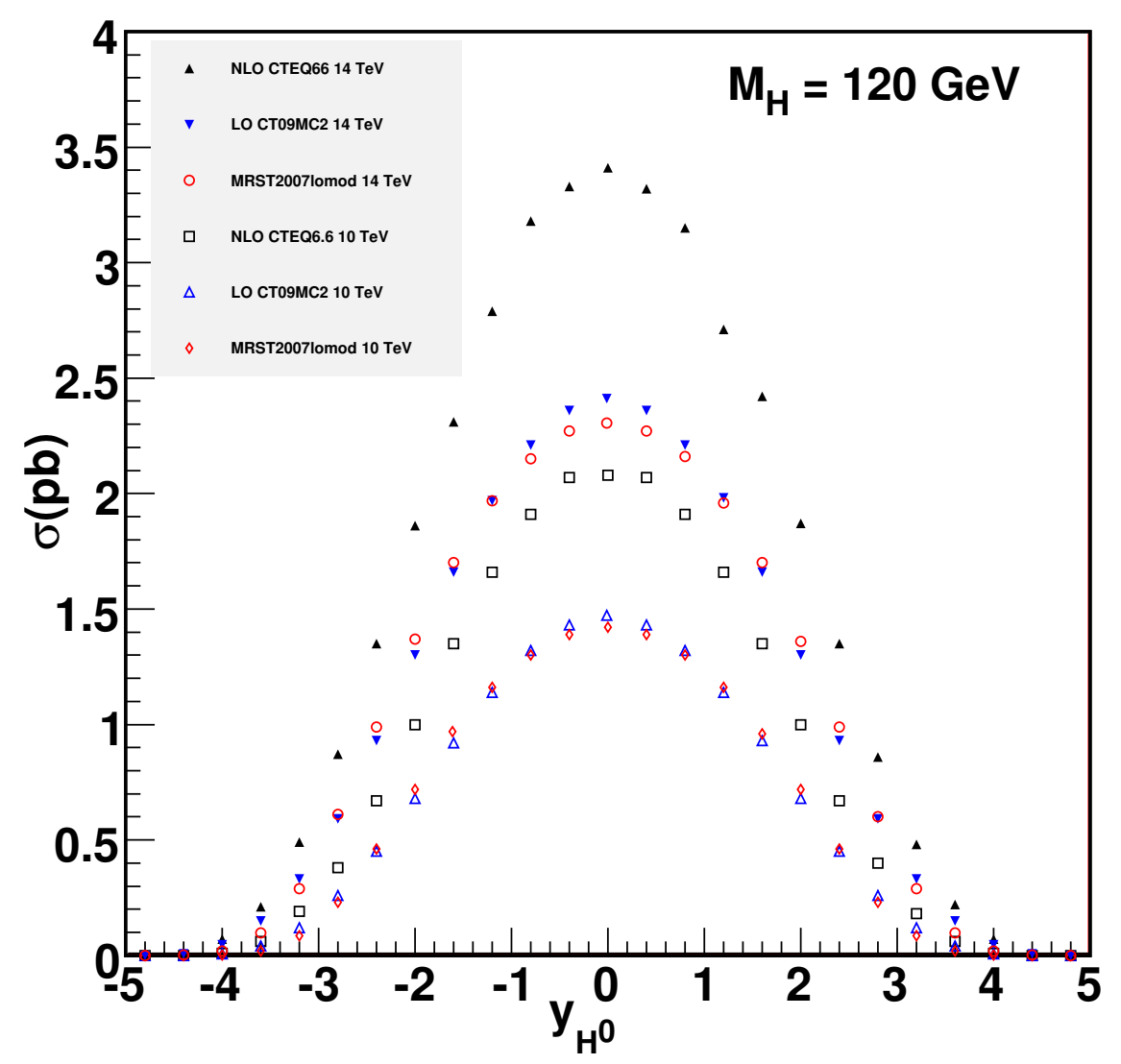

Figure 9. Same as figure 6, for the Higgs boson rapidity distribution at $\sqrt{s}=10$ and $14 \mathrm{TeV}$. To maintain legibility, the distribution for $\sqrt{s}=7 \mathrm{TeV}$ is not shown.

The K-factor for a given process is a useful shorthand which encapsulates the size of the NLO corrections to the lowest-order cross section. As discussed in appendix B, the K-factors are closer to unity when NLO PDFs are used for the LO calculations, and this is true as well for LO predictions using the CT09MC1 and CT09MC2 PDFs.

\section{Impact on the underlying event at the LHC}

Predictions for the underlying event at the LHC are most sensitive to the magnitude and shape of the low- $x$ gluon PDF, as the small- $x g g$ scattering into low- $p_{T}$ dijets makes up the bulk of the underlying event. As stated earlier, the LO gluon distribution is considerably larger at low $x$ than the NLO gluon. The multiple parton scattering models in the LO parton shower Monte Carlos have been tuned to this default LO gluon behavior. The CT09MC PDFs retain the low- $x$ behavior of the conventional LO gluon PDF and thus can be used with underlying event tunes similar to those derived for standard LO PDFs [15]. As an example, figure 18 shows PYTHIA [16] predictions for the charged particle transverse 


\section{$\mathrm{t} \overline{\mathrm{t}}$ mass distribution}

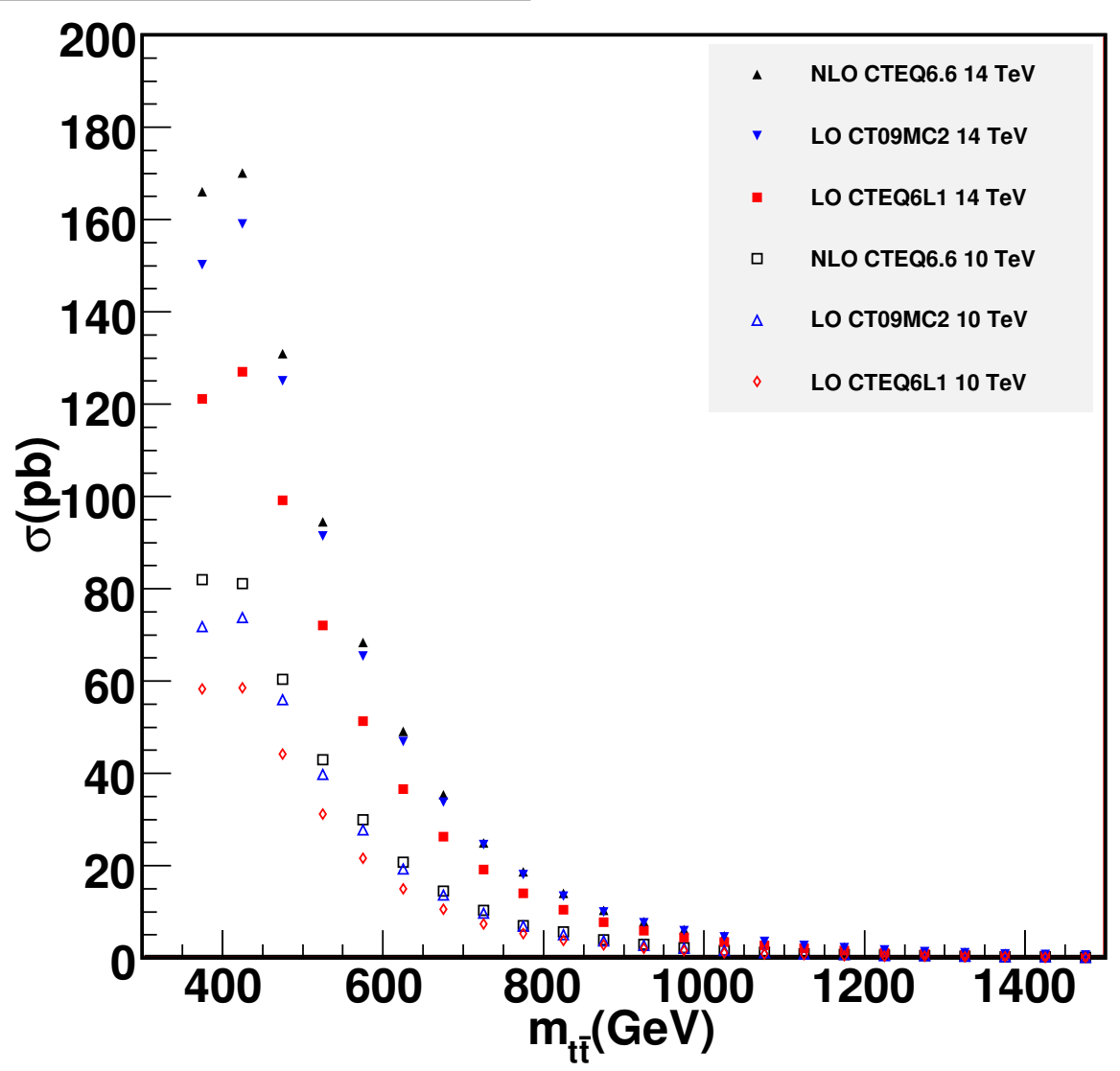

Figure 10. Predictions for the $t \bar{t}$ invariant mass distribution at the LHC $(\sqrt{s}=10$ and $14 \mathrm{TeV})$ in $50 \mathrm{GeV}$ mass bins, given at NLO using the CTEQ6.6M PDFs, and at LO using the CT09MC2 and CTEQ6L1 PDFs. The actual cross sections are shown.

momentum distribution in minimum bias events at CDF, obtained for PYTHIA Tune A [17] and CTEQ6L1, CT09MC1, and CT09MC2 PDFs. The two L0-MC PDFs lead to an equivalent description of the standard, Tune A with CTEQ6L1.

\section{Conclusion}

In this paper, we have generalized the conventional global QCD analysis to produce parton distributions optimized for simulations in event generators at leading order in perturbative QCD. This is done by combining the constraints due to existing hard-scattering experimental data with those from anticipated cross sections for key representative SM processes at the LHC (predicted by the NLO QCD theory) as a joint input to the global analysis. Results obtained from a few candidate PDF sets for LO event generators produced this way have been compared with those from other approaches. As compared to the conventional LO PDFs, the PDFs for leading-order Monte-Carlo event generators (LO-MC PDFs) de- 


\section{$\mathrm{t} \overline{\mathrm{t}}$ mass distribution}

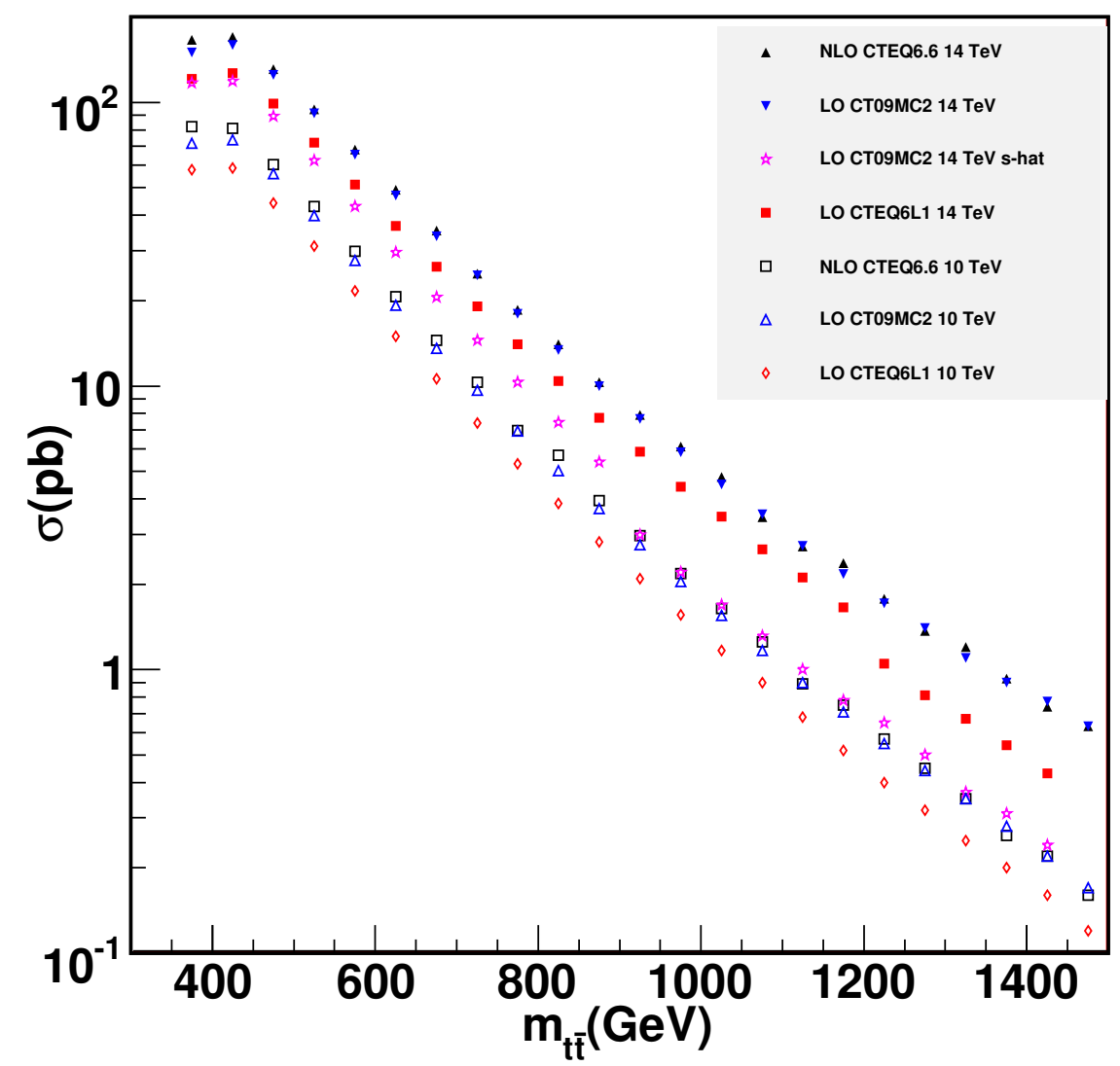

Figure 11. The same as in figure 10, on a semi-log scale. LO CT09MC2 predictions for the factorization scale $\mu=\sqrt{\widehat{s}}$ are also shown.

scribed here provide a better description for the normalization of the benchmark LHC cross sections, but, more importantly, for the shapes of these cross sections. In addition, we have performed validation studies to gauge the phenomenological impact of the CT09MC PDF sets and to locate any possible pathological behavior. Aside from the (desired) differences with the conventional LO PDFs noted in this paper, the effects are otherwise benign. In particular, the CT09MC PDF sets can be used with the underlying event tunes similar to those performed with CTEQ6L1. For the LHC processes discussed in section 6, we have checked kinematic properties of parton-level jets obtained with the new PDFs. After considering the individual $p_{T}$ and rapidity values of the jets, as well as variables sensitive to correlations between the jets, such as $m_{j j}, \Delta R(j, j)$, etc., no unexpected features were observed beyond the usual differences due to the choice of different PDF sets.

Given their good agreement with the anticipated LHC cross sections, the resulting PDFs are intended primarily for simulations for the LHC, and only using LO event generators. This study is our first attempt to develop such optimal PDFs. The discussed approach, and the choices made, are only representative of what can be achieved with 
Q value: $8 \mathrm{GeV}$, for parton: $\mathbf{g}$

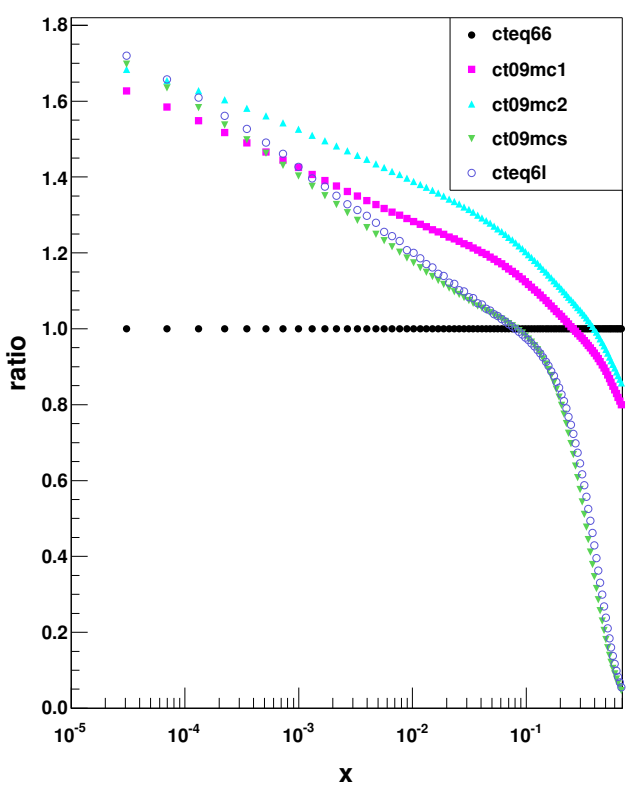

$Q$ value: $85 \mathrm{GeV}$, for parton: $\mathrm{g}$

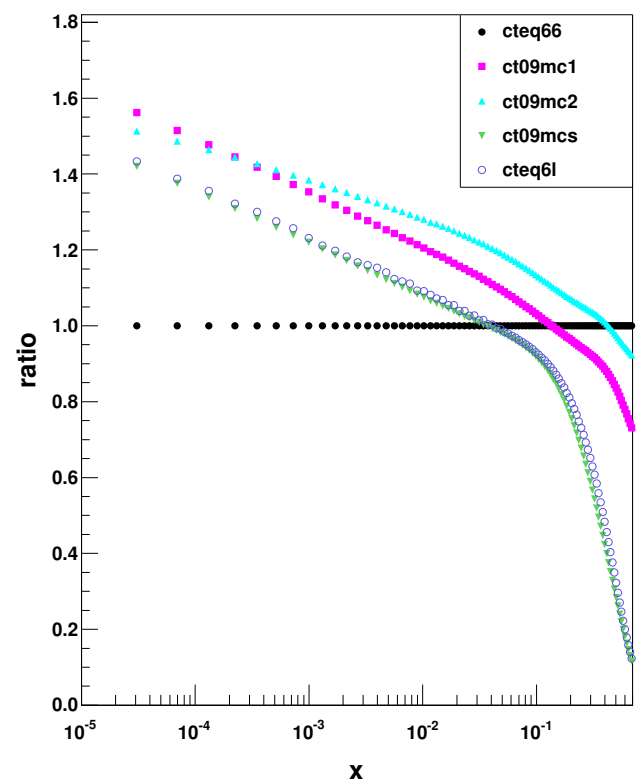

Figure 12. The ratio of gluon distributions from various LO PDFs to the gluon distribution from CTEQ6.6M at $Q$ values of 8 and $85 \mathrm{GeV}$.

$Q$ value: $8 \mathrm{GeV}$, for parton: $\mathrm{u}$

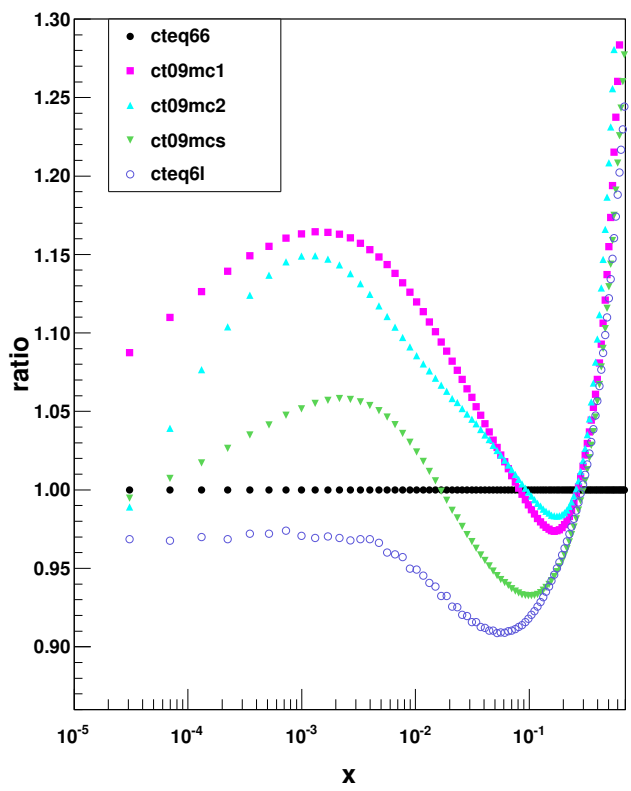

Q value: $85 \mathrm{GeV}$, for parton: u

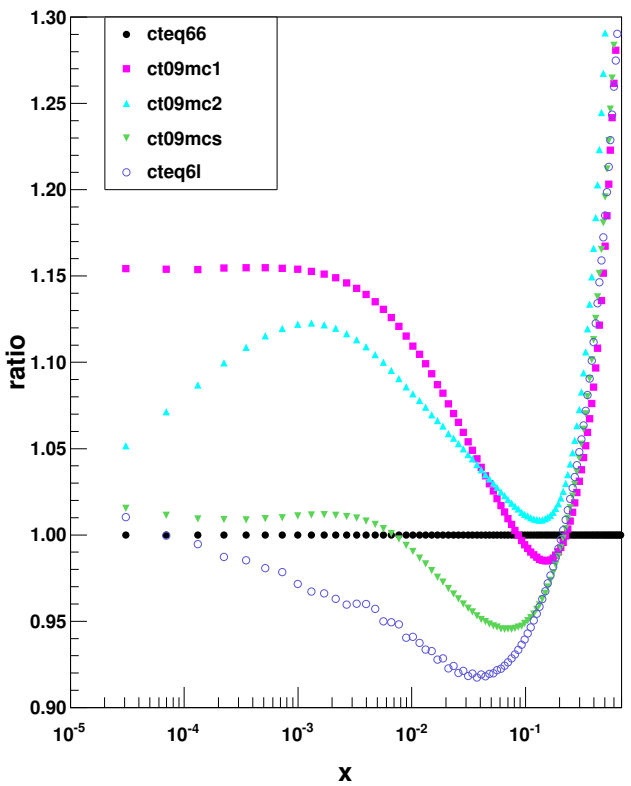

Figure 13. The ratio of the $u$ quark distributions from various LO PDFs to the $u$ quark distribution from CTEQ6.6M at $Q$ values of 8 and $85 \mathrm{GeV}$.

this method. Given the very nature of the LO event generators themselves, and the inherent uncertainties of any calculation done with LO matrix elements, it is the distinctive qualitative features of these PDFs described in earlier sections that matter the most. 
$Q$ value: $8 \mathrm{GeV}$, for parton: ub

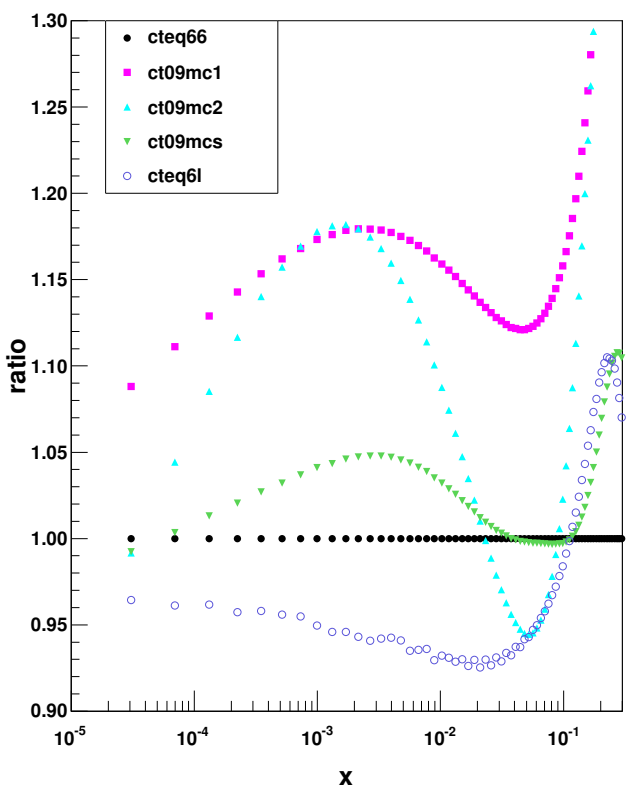

Q value: $85 \mathrm{GeV}$, for parton: ub

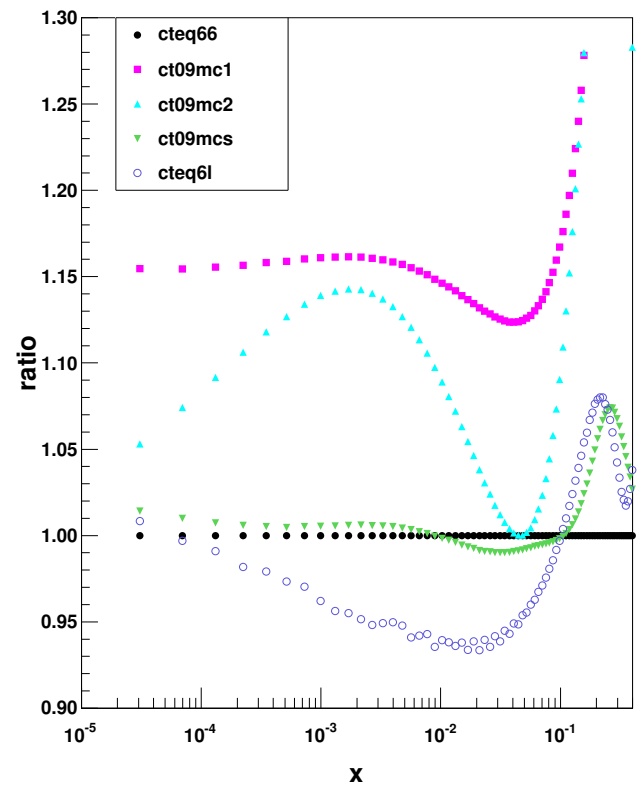

Figure 14. The ratio of the $\bar{u}$ distributions from various LO PDFs to the $\bar{u}$ distribution from CTEQ6.6M at $Q$ values of 8 and $85 \mathrm{GeV}$.

Q value: $8 \mathrm{GeV}$, for parton: $\mathrm{d}$

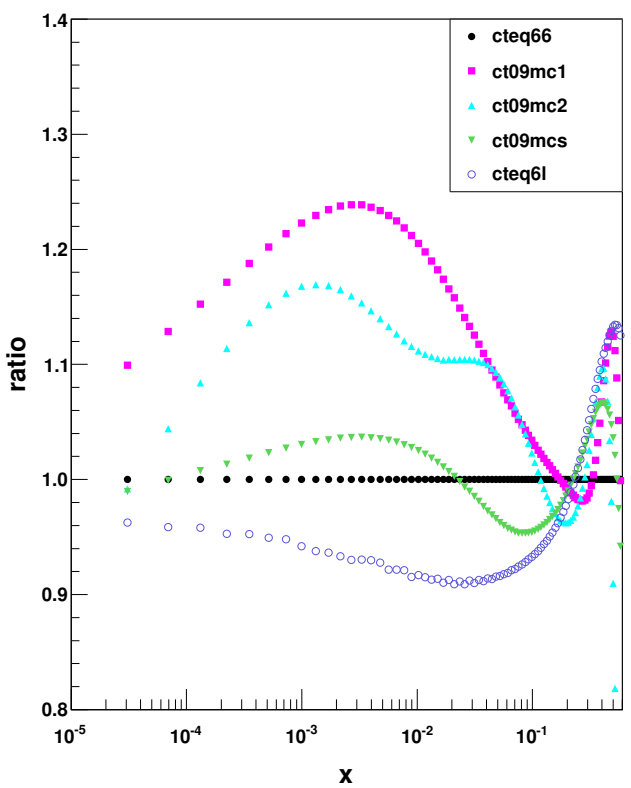

$Q$ value: $85 \mathrm{GeV}$, for parton: $\mathrm{d}$

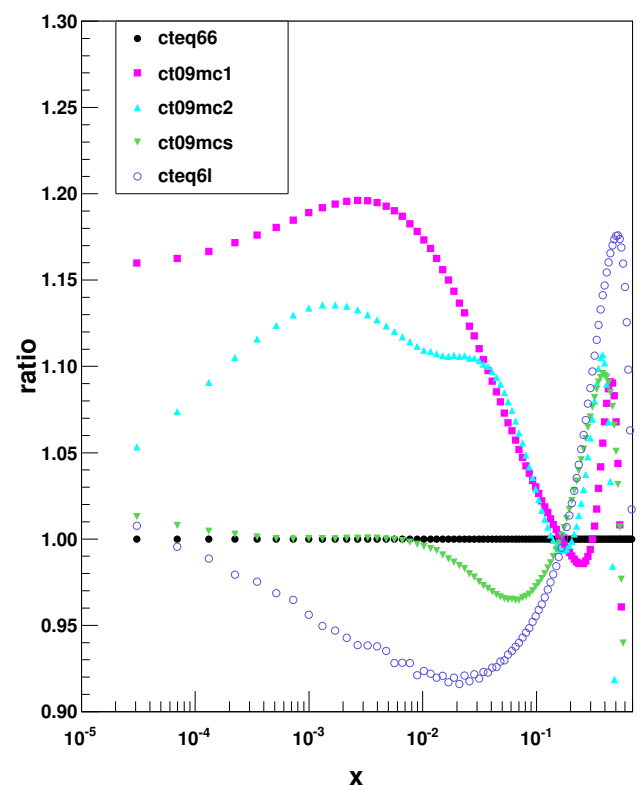

Figure 15. The ratio of the $d$ quark distributions from various LO PDFs to the $d$ quark distribution from CTEQ6.6M at $Q$ values of 8 and $85 \mathrm{GeV}$.

\section{In memoriam}

This paper would not have been possible without the insight and inspiration of our late colleague Wu-Ki Tung. 
$Q$ value: $8 \mathrm{GeV}$, for parton: $\mathrm{db}$

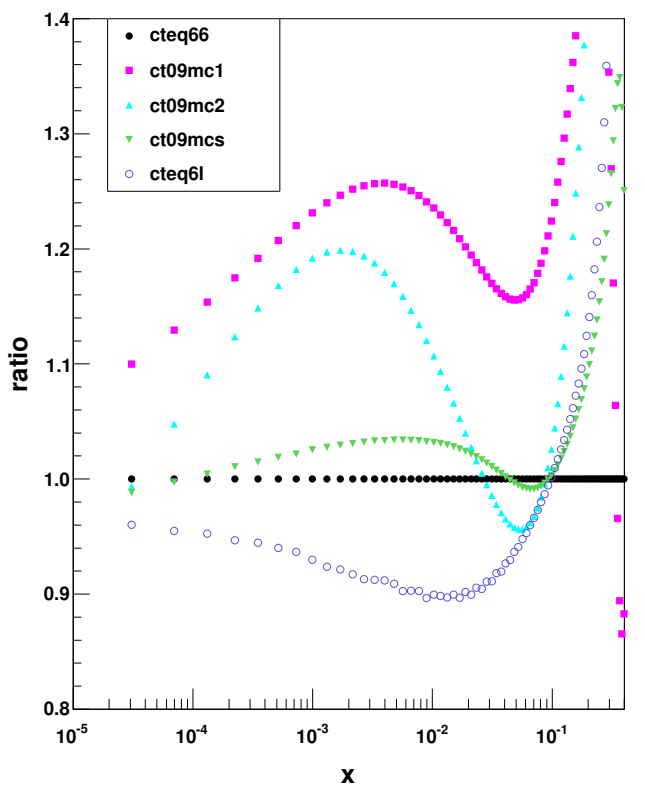

$Q$ value: $85 \mathrm{GeV}$, for parton: $\mathrm{db}$

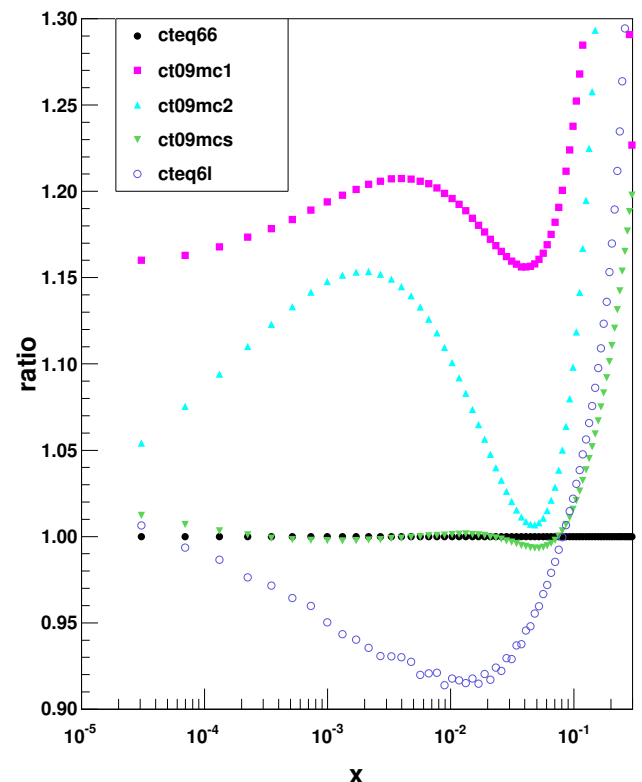

Figure 16. The ratio of the $\bar{d}$ distributions from various LO PDFs to the $\bar{d}$ distribution from CTEQ6.6M at $Q$ values of 8 and $85 \mathrm{GeV}$.

\section{Acknowledgments}

We would like to thank Albert de Roeck, Hannes Jung, Judith Katzy, Peter Skands, Torbjorn Sjostrand, Markus Warzinsky, and participants of the PDF4LHC meetings for useful discussions. This work was supported in part by the U.S. Department of Energy under grant DE-FG02-04ER41299; by the Fermilab Research Alliance, LLC, under Contract DE-AC02-76CH11359 with the United States Department of Energy; by the U.S. National Science Foundation under grant numbers PHY-0555545, PHY-0855561, and PHY-0757758; by the National Science Council of Taiwan under grant No. NSC-98-2112-M-133-002-MY3; by LHC Theory Initiative Travel Fellowship awarded by the U.S. National Science Foundation under grant PHY-0705862; and by Lightner-Sams Foundation. C.-P. Y. would also like to thank the hospitality of National Center for Theoretical Sciences in Taiwan and Center for High Energy Physics, Peking University, in China, where part of this work was done.

\section{A Production of a $W$-boson with $n$ partons in the LO-MC approach}

To study the impact of the LO-MC PDF sets on multi-parton configurations, of the kind commonly encountered in parton shower-matrix element matching, we have performed a parton-level calculation of $W^{+}+n$ parton cross sections $(n=0,1,2,3,4)$ at the LHC center-of-mass energy of $10 \mathrm{TeV}$ using MADGRAPH [14] and CTEQ6L1 and CT09MC2 PDF sets. The final-state colored partons were required to have transverse momenta $k_{T} \geq$ $10 \mathrm{GeV}$. The predicted cross sections are presented in table 3, broken down into different subprocess components and ranked by the relative size. For simplicity, we present only 

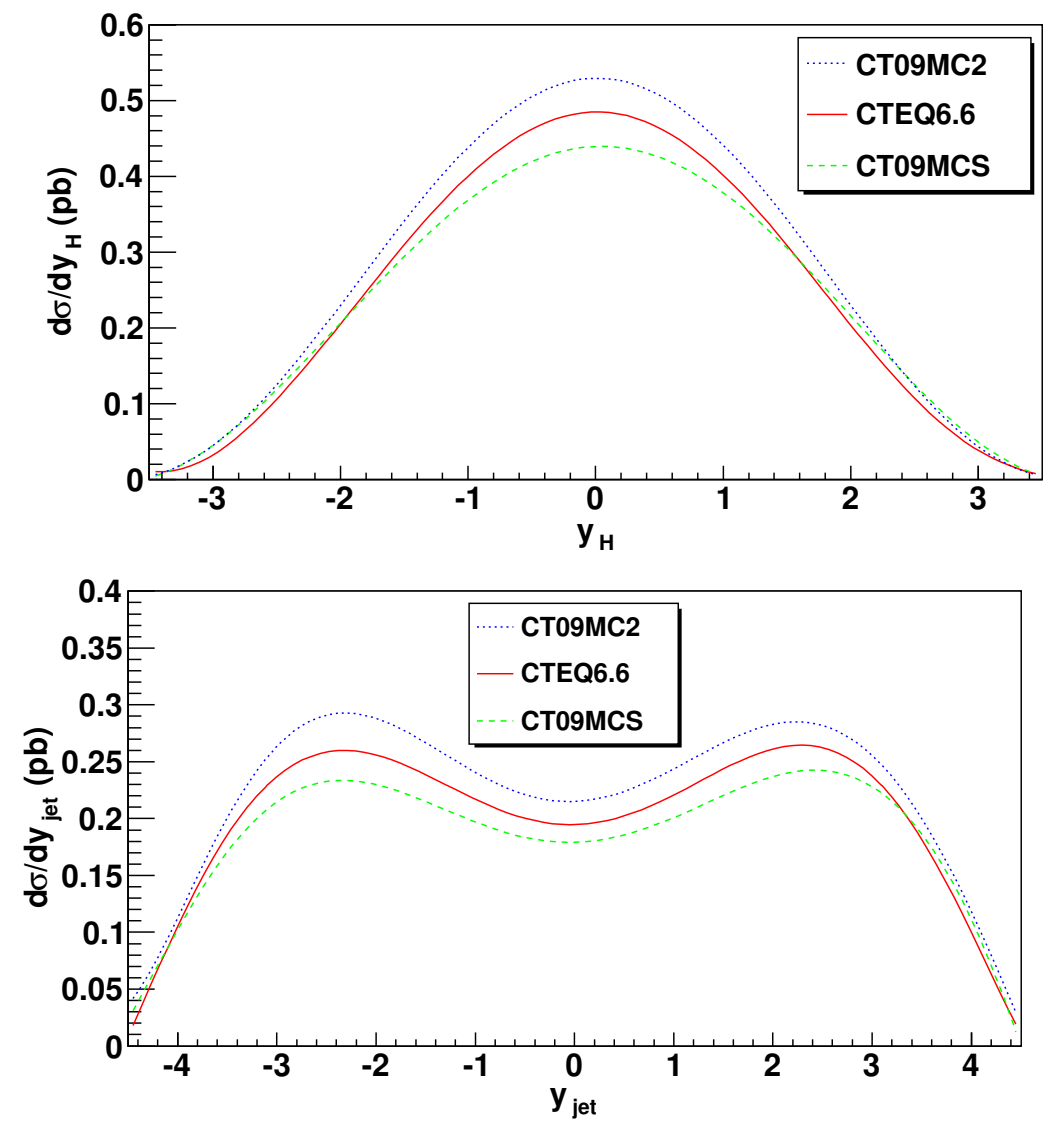

Figure 17. The rapidity distribution of $120 \mathrm{GeV}$ Higgs bosons produced through vector boson fusion at $\sqrt{s}=14 \mathrm{TeV}$ (top). Also shown is the distribution in the rapidity of the leading jet (bottom). NLO predictions are obtained with the CTEQ6.6M PDFs (solid curves), and LO predictions are for the CT09MCS (dashed curves) and CT09MC2 (dotted curves) PDFs. Here, the jets are separated by $\Delta R>0.4$ (with $R_{\text {sep }}=1.3$ ), and the transverse momentum and pseudorapidity of the jet satisfy $p_{T}>40 \mathrm{GeV} / c$ and $|\eta|<4.5$.

the results for up to two colored partons in the final state, and we compute the ratio $R_{\sigma}=\sigma(\mathrm{CT} 09 \mathrm{MC} 2) / \sigma(\mathrm{CTEQ} 6 \mathrm{~L} 1)$ for each scattering channel. While $R_{\sigma}$ is expected to vary between the different scattering channels, it is actually well represented by its average value in all channels, $R_{\sigma}=1.26$. A notable exception is the $g g$ initial state, with $R_{\sigma}=1.48$.

A similar study for subprocesses containing three or four colored partons in the final state reveals a similar pattern, but different values of $R_{\sigma}$. In these cases, $R_{\sigma}$ is equal to 1.34 for the total cross section, and $R_{\sigma}$ ranges from 1.48 to 1.77 for the gluon-gluon initial states.

The fact that $R_{\sigma}$ is different for different parton topologies will have some phenomenological impact. Color connections and parton types influence the parton shower: gluons and quarks have different Sudakov form factors, and color coherence limits the phase space for emissions. Thus, properties of jets resulting from a matched calculation based on the CT09MC2 PDFs are likely to be different from those based on CTEQ6L. The scale of these differences remains to be seen. 


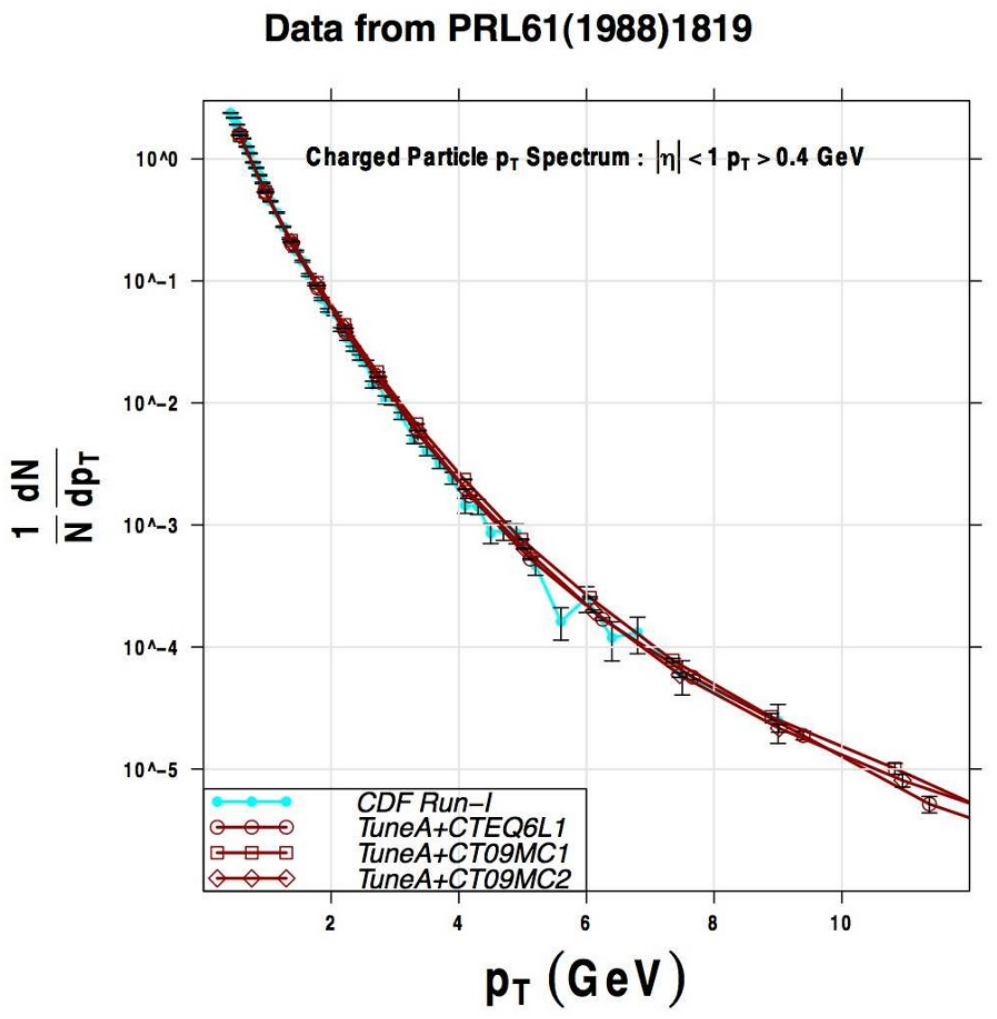

Figure 18. Predictions for the charged particle transverse momentum distribution in minimum bias events for CDF in the Tevatron Run 1 (1.8 TeV), using the CTEQ6L1, CT09MC1 and CT09MC2 PDFs.

The kinematics of partonic events is relatively unchanged between the two PDF sets, except for the distributions of the particles from the $W^{+}$boson decay. There is a tendency for colored partons to be more central with the CT09MC2 PDFs, but the difference is not significant. The change in shape of the rapidity distribution of the gauge boson has been discussed in the main text. The change in the distribution of the decay positron for various partonic multiplicities is shown in figure 19. Two features are notable in this figure. First, the CTEQ6L1 and CT09MC2 positron's rapidity distributions are different for all parton multiplicities $(n=0,1,2)$. Second, for a given PDF set, the distribution for $n=0$ is different from that for $n=1,2$. More detailed comparison will be deferred to future studies.

\section{B K-factors for LO-MC PDFs}

The K-factor, calculated as the ratio of the NLO to LO cross sections, depends on the choice of the renormalization/factorization scale, the PDFs used, and the kinematic region being considered. Even with the above caveats, it can be useful to define the K-factors for physics processes at the LHC [5]. Below we reproduce the table first shown in ref. [5] and then updated in the Les Houches 2007 proceedings, where we have included the K-factors 


\begin{tabular}{|l|l|l|l|}
\hline Subprocess & $\begin{array}{l}\sigma(\text { CTEQ6L1) } \\
(\mathrm{nb})\end{array}$ & $\begin{array}{l}\sigma(\mathrm{CT} 09 \mathrm{MC} 2) \\
(\mathrm{nb})\end{array}$ & Ratio $R_{\sigma}$ \\
\hline$u \bar{d} \rightarrow e^{+} \nu_{e}$ & 7.323 & 9.029 & 1.23 \\
\hline$u g \rightarrow e^{+} \nu_{e} d$ & 2.165 & 2.729 & 1.26 \\
\hline$u \bar{d} \rightarrow e^{+} \nu_{e} g$ & 1.760 & 2.207 & 1.25 \\
\hline$g \bar{d} \rightarrow e^{+} \nu_{e} \bar{u}$ & 0.835 & 1.130 & 1.35 \\
\hline$u g \rightarrow e^{+} \nu_{e} d g$ & 1.722 & 2.239 & 1.30 \\
\hline$g \bar{d} \rightarrow e^{+} \nu_{e} \bar{u} g$ & 0.546 & 0.751 & 1.38 \\
\hline$u \bar{d} \rightarrow e^{+} \nu_{e} g g$ & 0.325 & 0.416 & 1.28 \\
\hline$g g \rightarrow e^{+} \nu_{e} \bar{u} d$ & 0.138 & 0.204 & 1.48 \\
\hline$u u \rightarrow e^{+} \nu_{e} u d$ & 0.053 & 0.064 & 1.21 \\
\hline$u d \rightarrow e^{+} \nu_{e} d d$ & 0.038 & 0.047 & 1.24 \\
\hline$u \bar{d} \rightarrow e^{+} \nu_{e} d \bar{d}$ & 0.028 & 0.036 & 1.29 \\
\hline$u \bar{d} \rightarrow e^{+} \nu_{e} u \bar{u}$ & 0.026 & 0.033 & 1.27 \\
\hline$u \bar{s} \rightarrow e^{+} \nu_{e} d \bar{s}$ & 0.022 & 0.027 & 1.23 \\
\hline$u s \rightarrow e^{+} \nu_{e} d s$ & 0.022 & 0.027 & 1.23 \\
\hline$u \bar{u} \rightarrow e^{+} \nu_{e} \bar{u} d$ & 0.020 & 0.024 & 1.20 \\
\hline$u \bar{d} \rightarrow e^{+} \nu_{e} c \bar{c}$ & 0.019 & 0.024 & 1.26 \\
\hline$c \bar{d} \rightarrow e^{+} \nu_{e} \bar{u} c$ & 0.015 & 0.019 & 1.27 \\
\hline$u c \rightarrow e^{+} \nu_{e} u s$ & 0.013 & 0.016 & 1.23 \\
\hline$u c \rightarrow e^{+} \nu_{e} c d$ & 0.013 & 0.016 & 1.23 \\
\hline$d \bar{d} \rightarrow e^{+} \nu_{e} \bar{u} d$ & 0.012 & 0.016 & 1.33 \\
\hline$c \bar{u} \rightarrow e^{+} \nu_{e} \bar{u} s$ & 0.008 & 0.010 & 1.25 \\
\hline$u \bar{c} \rightarrow e^{+} \nu_{e} \bar{c} d$ & 0.007 & 0.009 & 1.29 \\
\hline $\bar{d} \bar{d} \rightarrow e^{+} \nu_{e} \bar{u} \bar{d}$ & 0.006 & 0.008 & 1.33 \\
\hline$u \bar{s} \rightarrow e^{+} \nu_{e} u \bar{c}$ & 0.006 & 0.008 & 1.33 \\
\hline Total & 15.12 & 19.09 & 1.26 \\
\hline & & & \\
\hline
\end{tabular}

Table 3. Breakdown of CTEQ6L1 and CT09MC2 cross sections and their ratios for different subprocesses of $W^{+}+n$ jet production $(n=0,1,2)$ at the LHC center-of-mass energy of $10 \mathrm{TeV}$.

using our LO-MC PDFs for processes at the LHC; a few of these processes were included as pseudodata in our global fit, while most were not. The result is shown in table 4 .

In most cases, the K-factors are smaller (closer to unity) when NLO PDFs are used for the LO calculations, and this is true as well for predictions using the LO-MC PDFs. The K-factor for $W$ production is less than 1 in this table: the $W$ pseudodata used in the LO-MC fit were generated with the CTEQ6.6M PDFs, which predict the LHC $W$ and $Z$ cross sections that are larger by 6-7\% than those based on CTEQ6 PDFs (used as NLO cross sections in the K-factor table) [12]. In this way, the effects of the variable flavor number heavy quark scheme used in the current NLO CTEQ PDF fits are effectively taken into account in the LO-MC formalism. The other quark-dominated process in the table below (vector boson fusion production of a $120 \mathrm{GeV}$ Higgs) also has a K-factor lower $(0.75$ compared to 0.85) when using the CTEQ6.6M PDFs for the NLO calculation, rather than the CTEQ6 PDFs. The K-factors for the other processes are nearly the same for CTEQ6M as for CTEQ6.6M. 


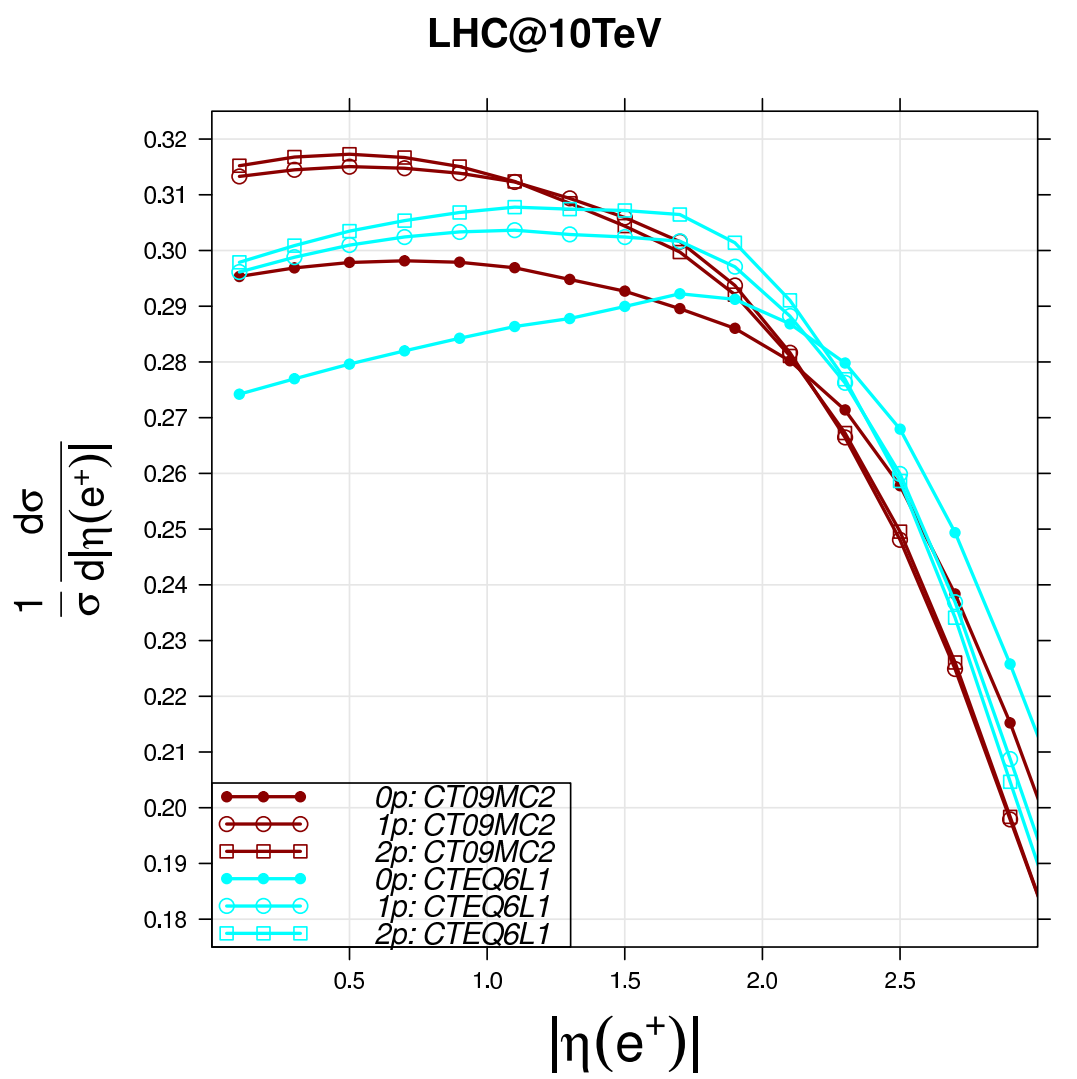

Figure 19. Distribution of positron pseudorapidity $\left|\eta\left(e^{+}\right)\right|$for various partonic $p$ multiplicities in the case of $W^{+}+n$ jets $(n=0,1,2)$ production at the LHC (with a center of mass energy of $10 \mathrm{TeV}$ ), for CTEQ6L1 and CT09MC2 PDF sets. The partonic jets are defined with $k_{T} \geq 10 \mathrm{GeV}$.

\begin{tabular}{|l|l|l|c|c|c|c|c|c|c|}
\hline & \multicolumn{2}{|l|}{ Fact. scales } & \multicolumn{3}{|c|}{ Tevatron K-factor } & \multicolumn{4}{|c|}{ LHC K-factor } \\
Process & $\mu_{0}$ & $\mu_{1}$ & $\mathcal{K}\left(\mu_{0}\right)$ & $\mathcal{K}\left(\mu_{1}\right)$ & $\mathcal{K}^{\prime}\left(\mu_{0}\right)$ & $\mathcal{K}\left(\mu_{0}\right)$ & $\mathcal{K}\left(\mu_{1}\right)$ & $\mathcal{K}^{\prime}\left(\mu_{0}\right)$ & $\mathcal{K}^{\prime \prime}\left(\mu_{0}\right)$ \\
\hline$W$ & $m_{W}$ & $2 m_{W}$ & 1.33 & 1.31 & 1.21 & 1.15 & 1.05 & 1.15 & 0.95 \\
$W+1$ jet & $m_{W}$ & $p_{T}^{\text {jet }}$ & 1.42 & 1.20 & 1.43 & 1.21 & 1.32 & 1.42 & 0.99 \\
$W+2$ jets & $m_{W}$ & $p_{T}^{\text {jet }}$ & 1.16 & 0.91 & 1.29 & 0.89 & 0.88 & 1.10 & 0.90 \\
$W W+1$ jet $[18,19]$ & $m_{W}$ & $2 m_{W}$ & 1.19 & 1.37 & 1.26 & 1.33 & 1.40 & 1.42 & 1.10 \\
$t \bar{t}$ & $m_{t}$ & $2 m_{t}$ & 1.08 & 1.31 & 1.24 & 1.40 & 1.59 & 1.19 & 1.09 \\
$t \bar{t}+1$ jet $[20]$ & $m_{t}$ & $2 m_{t}$ & 1.13 & 1.43 & 1.37 & 0.97 & 1.29 & 1.10 & 0.85 \\
$b \bar{b}$ & $m_{b}$ & $2 m_{b}$ & 1.20 & 1.21 & 2.10 & 0.98 & 0.84 & 2.51 & - \\
Higgs & $m_{H}$ & $p_{T}^{\text {jet }}$ & 2.33 & - & 2.33 & 1.72 & - & 2.32 & 1.43 \\
Higgs via VBF & $m_{H}$ & $p_{T}^{\text {jet }}$ & 1.07 & 0.97 & 1.07 & 1.23 & 1.34 & 0.85 & 0.83 \\
Higgs +1 jet & $m_{H}$ & $p_{T}^{\text {jet }}$ & 2.02 & 1.46 & 2.13 & 1.47 & 1.24 & 1.90 & 1.33 \\
Higgs +2 jets $[21]$ & $m_{H}$ & $p_{T}^{\text {jet }}$ & - & - & - & 1.15 & - & - & 1.13 \\
\hline
\end{tabular}

Table 4. K-factors for various processes at the LHC (at $14 \mathrm{TeV}$ ) calculated using a selection of input parameters. In all cases, for NLO calculations, the CTEQ6M PDF set is used. For LO calculations, $\mathcal{K}$ uses the CTEQ6L1 set, whilst $\mathcal{K}^{\prime}$ uses the same PDF set, CTEQ6M, as at NLO, and $\mathcal{K}^{\prime \prime}$ uses the LO-MC (2-loop) PDF set CT09MC2. For Higgs+1 or 2 jets, a jet cut of $40 \mathrm{GeV} / c$ and $|\eta|<4.5$ has been applied. A cut of $p_{T}^{\text {jet }}>20 \mathrm{GeV} / c$ has been applied to the $t \bar{t}+$ jet process, and a cut of $p_{T}^{\text {jet }}>50 \mathrm{GeV} / c$ to the $W W+$ jet process. In the $W$ (Higgs) +2 jets process, the jets are separated by $\Delta R>0.4$ (with $R_{\text {sep }}=1.3$ ), whilst the vector boson fusion (VBF) calculations are performed for a Higgs boson of mass $120 \mathrm{GeV}$. In each case the value of the $\mathrm{K}$-factor is compared at two often-used scale choices, $\mu_{0}$ and $\mu_{1}$. 
Open Access. This article is distributed under the terms of the Creative Commons Attribution Noncommercial License which permits any noncommercial use, distribution, and reproduction in any medium, provided the original author(s) and source are credited.

\section{References}

[1] S. Frixione and B.R. Webber, Matching NLO QCD computations and parton shower simulations, JHEP 06 (2002) 029 [hep-ph/0204244] [SPIRES].

[2] P. Nason, A new method for combining NLO QCD with shower Monte Carlo algorithms, JHEP 11 (2004) 040 [hep-ph/0409146] [SPIRES].

[3] S. Frixione, P. Nason and C. Oleari, Matching NLO QCD computations with Parton Shower simulations: the POWHEG method, JHEP 11 (2007) 070 [arXiv: 0709. 2092] [SPIRES].

[4] J.C. Collins and X.-m. Zu, Parton distribution functions suitable for Monte-Carlo event generators, JHEP 06 (2002) 018 [hep-ph/0204127] [SPIRES].

[5] J.M. Campbell, J.W. Huston and W.J. Stirling, Hard interactions of quarks and gluons: a primer for LHC physics, Rept. Prog. Phys. 70 (2007) 89 [hep-ph/0611148] [SPIRES].

[6] T. Sjöstrand, S. Mrenna and P.Z. Skands, A brief introduction to PYTHIA 8.1, Comput. Phys. Commun. 178 (2008) 852 [arXiv:0710.3820] [SPIRES].

[7] A. Sherstnev and R.S. Thorne, Parton distributions for LO generators, Eur. Phys. J. C 55 (2008) 553 [arXiv:0711.2473] [SPIRES].

[8] A. Sherstnev and R.S. Thorne, Different PDF approximations useful for LO Monte Carlo generators, arXiv:0807.2132 [SPIRES].

[9] H. Jung et al., PDF 4MC, online at http://indico.cern.ch/getFile.py/access?contribId=14 \&sessionId $=0 \&$ resId $=0 \&$ materialId $=$ slides \&confId $=36364$.

[10] F. Maltoni, T. McElmurry, R. Putman and S. Willenbrock, Choosing the factorization scale in perturbative $Q C D$, hep-ph/0703156 [SPIRES].

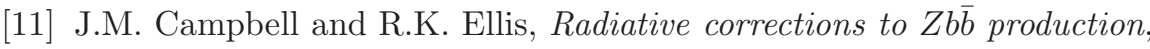
Phys. Rev. D 62 (2000) 114012 [hep-ph/0006304] [SPIRES].

[12] P.M. Nadolsky et al., Implications of CTEQ global analysis for collider observables, Phys. Rev. D 78 (2008) 013004 [arXiv:0802.0007] [SPIRES].

[13] J. Pumplin et al., New generation of parton distributions with uncertainties from global QCD analysis, JHEP 07 (2002) 012 [hep-ph/0201195] [SPIRES].

[14] J. Alwall et al., MadGraph/MadEvent v4: the new web generation, JHEP 09 (2007) 028 [arXiv:0706.2334] [SPIRES].

[15] M. Warshinsky, private communication.

[16] T. Sjöstrand, S. Mrenna and P.Z. Skands, PYTHIA 6.4 physics and manual, JHEP 05 (2006) 026 [hep-ph/0603175] [SPIRES].

[17] http://www.phys.ufl.edu/ rfield/cdf/tunes/py_tuneA.html.

[18] S. Dittmaier, S. Kallweit and P. Uwer, $N L O Q C D$ corrections to $W W+$ jet production at hadron colliders, Phys. Rev. Lett. 100 (2008) 062003 [arXiv:0710.1577] [SPIRES].

[19] J.M. Campbell, R. Keith Ellis and G. Zanderighi, Next-to-Leading Order predictions for $W W+1$ jet distributions at the LHC, JHEP 12 (2007) 056 [arXiv:0710.1832] [SPIRES]. 
[20] S. Dittmaier, P. Uwer and S. Weinzierl, Hadronic top-quark pair production in association with a hard jet at Next-to-Leading Order QCD: phenomenological studies for the Tevatron and the LHC, Eur. Phys. J. C 59 (2009) 625 [arXiv:0810.0452] [SPIRES].

[21] J.M. Campbell, R.K. Ellis and G. Zanderighi, Next-to-Leading Order Higgs +2 jet production via gluon fusion, JHEP 10 (2006) 028 [hep-ph/0608194] [SPIRES]. 